Research Article

\title{
Vibration Bandgaps of Piezoelectric Metamaterial Plate with Local Resonators for Vibration Energy Harvesting
}

\author{
Zhongsheng Chen $\mathbb{D}^{1,2}$ Jing He $\mathbb{D}^{1},{ }^{1}$ and Gang Wang $\mathbb{D}^{3}$ \\ ${ }^{1}$ College of Electrical \& Information Engineering, Hunan University of Technology, Zhuzhou 417002, China \\ ${ }^{2}$ Science and Technology on Integrated Logistics Support Laboratory, National University of Defense Technology, \\ Changsha 410073, China \\ ${ }^{3}$ State Key Laboratory of Advanced Design and Manufacturing for Vehicle Body, Hunan University, Changsha 410082, China \\ Correspondence should be addressed to Zhongsheng Chen; chenzs_hut@sina.com and Jing He; hejing@263.net
}

Received 3 February 2019; Revised 25 March 2019; Accepted 10 April 2019; Published 24 June 2019

Academic Editor: Carlo Trigona

Copyright (C) 2019 Zhongsheng Chen et al. This is an open access article distributed under the Creative Commons Attribution License, which permits unrestricted use, distribution, and reproduction in any medium, provided the original work is properly cited.

\begin{abstract}
Embedded wireless sensing networks (WSNs) provide effective solutions for structural health monitoring (SHM), where how to provide long-term electric power is a bottle-neck problem. Piezoelectric vibration energy harvesting (PVEH) has been widely studied to realize self-powered WSNs due to piezoelectric effect. Structural vibrations are usually variable and exist in the form of elastic waves, so cantilever-like harvesters are not appropriate. In this paper, one kind of two-dimensional (2D) piezoelectric metamaterial plates with local resonators (PMP-LR) is investigated for structural vibration energy harvesting. In order to achieve low-frequency and broadband PVEH in SHM, it is highly necessary to study dynamic characteristics of PMP-LR, particularly bandgaps. Firstly, an analytical model is developed based on the Kirchhoff plate theory, and modal analysis is done by using the Rayleigh-Ritz method. Then, effects of geometric and material parameters on vibration bandgaps are analyzed by finite elementbased simulations. In the end, experiments are carried out to validate the simulated results. The results demonstrate that the location of bandgaps can be easily adjusted by the design of local resonators. Therefore, the proposed method will provide an effective tool for optimizing local resonators in PMP-LR.
\end{abstract}

\section{Introduction}

Structural health monitoring (SHM) is the process of detecting the states of aerospace, civil, and mechanical infrastructure during their lives [1]. SHM aims to improve the safety, reliability, and/or ownership costs of engineering systems and structures by autonomously monitoring their conditions and detecting incipient damages before they reach a critical state. In order to achieve it, condition-based damage assessment ways have been utilized to replace qualitative visual inspection and time-based maintenance methods. Most monitoring systems for SHM are wired in a wide area or in a harsh environment, resulting in both economical and practical limitations. Thanks to the development of MEMS technologies, many microsensors can be embedded into engineering structures and useful signals can be transmitted wirelessly, such as acceleration, strain, and so on. Thus, embedded wireless sensing network (WSN) provides effective solutions for SHM. However, a major concern with each embedded WSN is their long-term sources of power [2]. Nowadays, the most suitable solution to extend the life is to harvest the environmental energy to generate electrical energy, which is called energy harvesting [3].

As one of energy harvesting technologies, piezoelectric vibration energy harvesting can harvest electrical energy from mechanical vibrations based on the direct piezoelectric effect [4]. Piezoelectric energy harvesting is superior to other vibration-to-electricity conversion mechanisms due to large energy density and ease of implementation. Thus piezoelectric vibration energy harvesting (PVEH) has been widely studied to realize self-powered WSNs $[5,6]$. Most PVEH devices take a form of cantilever, which has some drawbacks 
from the viewpoints of SHM systems. Firstly, it requires an extra space to hold a bulky proof mass and clamping part. Secondly, it is difficult to effectively harvest low-frequency vibrations due to the length limitation. For SHM, the disadvantages of the cantilever motivated the study of piezoelectric patch-based energy harvesters integrated to the structures to convert vibration energy of their host structure into electrical energy. Piezoelectric patches can be feasible especially for thin structures used in the fields of aerospace, automotive, and marine applications. Lee and Youn presented a design and experimental verification methodology for piezoelectric energy harvesting skin, which was optimized by two steps [7]. Later, Yoon et al. developed an electromechanically coupled analytical model of PEH skin considering the inertia and stiffness effects of a piezoelectric patch [8]. Erturk presented analytical formulation for energy harvesting with piezoelectric patches from surface fluctuations of large and high impedance structures [9]. Aridogan et al. [10] derived analytical closed-form expressions of piezoelectric patch-based energy harvesters structurally integrated to fully clamped plates. However, those works mostly focus on a single piezoelectric patch, and vibration propagations on the thin plate are often less considered. Structural vibrations are usually variable and exist in the form of elastic waves. Therefore, there is a need to control the propagation of elastic waves so that vibrations can be concentrated in a local area to be captured effectively. Otherwise, compact and broadband vibration energy harvesting in structures cannot be achieved. The solution of this problem is to develop innovative energy harvesting structures.

In recent years, artificial materials and structures with periodic modulations in their physical properties have attracted significant attention due to their unusual acoustic and elastic properties. Due to material and geometrical discontinuities in the individual cells of periodic structures, waves only propagate along the periodic cells within specific frequency bands, while these waves are completely blocked within other frequency bands called "bandgaps." That is to say, vibration energy within bandgaps may be localized in the cells, which is beneficial for energy harvesting. Thus, we can design optimal bandgaps to meet the challenges of low-frequency and broadband vibration energy harvesting in SHM. Periodic structural configurations can help to design both phononic crystals and metamaterials. Gonella et al. [11] discussed the interplay between phononic bandgaps and piezoelectric microstructures for energy harvesting. Chen et al. [12] explored one-dimensional phononic piezoelectric cantilever beams to widen the resonant bandwidth of a harvester. Wu et al. first used a phononic crystal to harvest acoustic energy [13]. But for phononic crystal, the lattice constant is required to be on the order of the wavelength so that its length scale is often large. Liu et al. [14] firstly proposed the concept of acoustic metamaterial which provided a promising solution for the length-scale problem of bandgap materials. Structural unit sizes of acoustic metamaterials are much smaller than the acoustic wavelength, and each unit cell has its own mechanical oscillator. Due to the local resonant behaviors of these auxiliary oscillators, bandgaps can be created in the low-frequency regime. Therefore, locally resonant metamaterials will provide an effective way for harvesting structural vibrations. Sugino et al. presented analytical and numerical investigations on a one-dimensional locally resonant piezoelectric metastructure with segmented electrodes under transverse vibrations [15]. Kherraz et al. studied numerically and experimentally the locally resonant bandgap in a homogeneous piezoelectric plate covered by a $1 \mathrm{D}$ periodic array of thin electrodes connected to inductive shunts [16]. So far, a small amount of research has been done on PVEH based on piezoelectric metamaterial plates.

The strategy used for vibration energy harvesting based on piezoelectric metamaterial plates includes two steps. The first one is to optimize the bandgaps so that targeted broadband and low-frequency vibrations can be localized in some unit cells based on locally resonant mechanism. In this case, these local resonators act as local absorbers of mechanical vibrations and electric charges can be generated from the strain field based on the piezoelectric effect. The other one is to connect these unit cells to interface circuits, which are then optimized to transform and store the harvested power based on the electromechanically coupled model of the whole system. This paper will focus on the first step, and one kind of two-dimensional (2D) piezoelectric metamaterial plates is investigated for vibration energy harvesting. Periodic square holes are cut off from a base plate, which are then covered by piezoelectric patches equipped with a locally resonant mass. In nature, the local resonator acts as an absorber of mechanical vibrations. This special periodic structure is called a piezoelectric metamaterial plate with local resonators (PMP-LR) in this paper. In order to achieve low-frequency and broadband PVEH in SHM, one needs to study its dynamic characteristics, in particular the bandgaps. Therefore, this study aims to develop an electromechanically coupled analytical model based on the Kirchhoff plate theory to analyze vibration bandgaps of the PMP-LR, which are strongly related to its geometric and material properties. Thus, it is necessary to investigate the effects of these key parameters for structural optimization. The remainder of this paper is organized as follows. In Section 2, the electromechanical model of a PMP-LR is built and modal analysis of the PMPLR is done based the Rayleigh-Ritz method. Vibration bandgaps of the PMP-LR are derived based on the Bloch theorem in Section 3. In Section 4, finite element-based vibration simulations are done to analyze effects of geometric and material parameters on vibration bandgaps and experiments are carried out to validate simulated results. Finally, the conclusions of this work are summarized in Section 6.

\section{Analytical Modeling of a PMP-LR}

2.1. Configuration. In this paper, basic configuration of the $2 \mathrm{D}$ piezoelectric metamaterial plate is shown in Figure 1. A thin rectangular plate is used as the base structure, within which periodic square holes with the same size are cut off. 


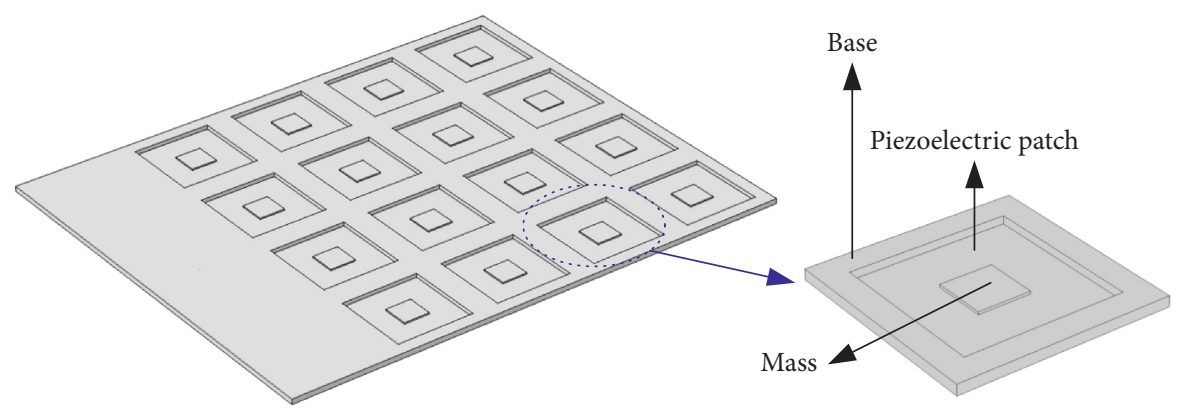

Figure 1: Basic configuration of a PMP-LR.

Then, each square hole is covered with a piezoelectric patch that supports a small square mass. The piezoelectric patch and the mass form a local resonator. Furthermore, each unit cell of the 2D PMP-LR consists of a base plate-like structure with a square hole, a piezoelectric patch, and a small square mass.

Vibrations of the PMP-LR under excitations will propagate along the plane of the plate. Due to the existence of local resonators, some vibrations within specific frequency bands cannot propagate through the periodic cells, leading to the bandgaps. In this case, vibration energies will be localized in some cells, which then can be converted into electricity through piezoelectric patches. In practice, the key is to understand vibration bandgaps of the PMP-LR so that we can optimally design the PMP-LR to meet the practical requirements.

\subsection{Dynamic Modeling of the PMP-LR Based on Hamilton's} Principle. Since the piezoelectric patches are typically manufactured as a thin plate, the PMP-LR can be modeled as a 2D Kirchhoff plate. According to the classical Kirchhoff plate theory [8], the PMP-LR will satisfy the following assumptions: (1) the thickness of the PMP-LR is small compared to its other dimensions; (2) the transverse deflection is small compared to the thickness of the PMP-LR; (3) the influence of transverse shear deformation is neglected; and (4) the effect of rotary inertia is neglected. The PMP-LR is assumed to be fixed at the left, and its geometrical dimensions are shown in Figure 2. The length and width of the aluminum base structure are denoted as $b$ and $a$ respectively. The side lengths of the cell and the piezoelectric patch are denoted as $l_{\mathrm{c}}$ and $l_{\mathrm{p}}$, respectively. Thickness of the base structure and the piezoelectric patch are $h_{\mathrm{s}}$ and $h_{\mathrm{p}}$, respectively. The plane of the PMP-LR corresponds to the $x-y$ plane, and the $z$-axis is perpendicular to the $x-y$ plane which is not displayed in Figure 2. A vibration excitation perpendicular to the PMP-LR is assumed to act at the location of $\left(x_{0}, y_{0}\right)$, and then transverse displacements will be generated, which are denoted as $w=w(x, y, t)$.

2.2.1. Constitutive Equations of the Base Structure and Piezoelectric Patch. Based on the Kirchhoff plate theory, the transverse shear strains are negligible. Then, the middle layer of the base structure is considered as the middle surface. In this case, the relationship between the strain field and

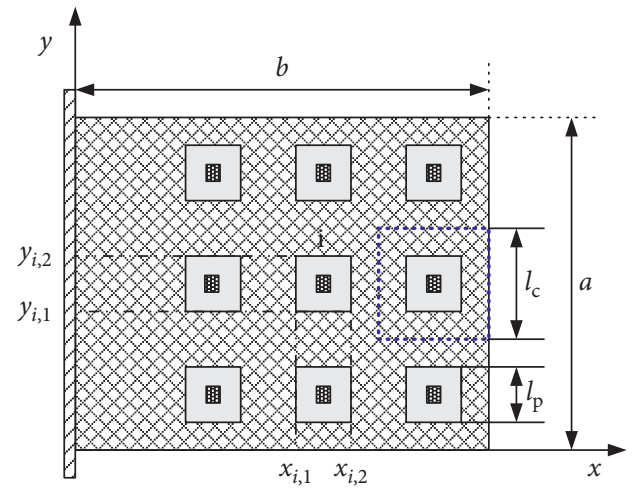

Figure 2: Geometrical dimensions of the PMP-LR.

transverse displacement of the base structure can be represented as

$$
\left\{\begin{array}{l}
\varepsilon_{x}^{s}=-z \frac{\partial^{2} w(x, y, t)}{\partial x^{2}}, \\
\varepsilon_{y}^{s}=-z \frac{\partial^{2} w(x, y, t)}{\partial y^{2}}, \\
\gamma_{x y}^{s}=-2 z \frac{\partial^{2} w(x, y, t)}{\partial x \partial y},
\end{array}\right.
$$

where $\varepsilon_{x}^{\mathrm{s}}, \varepsilon_{y}^{\mathrm{s}}$, and $\gamma_{x y}^{\mathrm{s}}$ are the strains in the $x, y$, and $x-y$ directions, respectively, and the superscript of " $\mathrm{s}$ " denotes the base structure.

Next, the constitutive equations for the base structure layer are reduced to

$$
\left[\begin{array}{c}
\sigma_{x}^{\mathrm{s}} \\
\sigma_{y}^{\mathrm{s}} \\
\tau_{x y}^{\mathrm{s}}
\end{array}\right]=\frac{E_{\mathrm{s}}}{1-\mu_{\mathrm{s}}^{2}}\left[\begin{array}{ccc}
1 & \mu_{\mathrm{s}} & 0 \\
\mu_{\mathrm{s}} & 1 & 0 \\
0 & 0 & \frac{1-\mu_{s}}{2}
\end{array}\right]\left[\begin{array}{c}
\varepsilon_{x}^{\mathrm{s}} \\
\varepsilon_{y}^{\mathrm{s}} \\
\gamma_{x y}^{\mathrm{s}}
\end{array}\right],
$$

where $E_{\mathrm{s}}$ is Young's modulus, $\mu_{\mathrm{s}}$ is Poisson's ratio of the base structural layer, and $\sigma_{x}^{\mathrm{s}}, \sigma_{y}^{\mathrm{s}}$, and $\tau_{x y}^{\mathrm{s}}$ are the stresses in the $x$, $y$, and $x-y$ directions, respectively.

By combining equations (1) and (2), the relationship between the stress and transverse displacement of the base structural layer can be obtained as 


$$
\left\{\begin{array}{l}
\sigma_{x}^{\mathrm{s}}=-\frac{E_{\mathrm{s}} z}{1-\mu_{\mathrm{s}}^{2}}\left(\frac{\partial^{2} w(x, y, t)}{\partial x^{2}}+\mu_{\mathrm{s}} \frac{\partial^{2} w(x, y, t)}{\partial y^{2}}\right) \\
\sigma_{y}^{\mathrm{s}}=-\frac{E_{\mathrm{s}} z}{1-\mu_{\mathrm{s}}^{2}}\left(\mu_{\mathrm{s}} \frac{\partial^{2} w(x, y, t)}{\partial x^{2}}+\frac{\partial^{2} w(x, y, t)}{\partial y^{2}}\right) \\
\tau_{x y}^{\mathrm{s}}=-\frac{E_{\mathrm{s}} z}{1+\mu_{\mathrm{s}}} \frac{\partial^{2} w(x, y, t)}{\partial x \partial y}
\end{array}\right.
$$

Furthermore, a piezoelectric patch can generally be considered as a transversely isotropic material. Then, the piezoelectric patches can also be assumed to be a thin plate. Similarly, the relationship between the strain field and transverse displacement of a piezoelectric patch can be represented as

$$
\left\{\begin{array}{l}
\varepsilon_{x}^{\mathrm{p}}=-z \frac{\partial^{2} w(x, y, t)}{\partial x^{2}}, \\
\varepsilon_{y}^{\mathrm{p}}=-z \frac{\partial^{2} w(x, y, t)}{\partial y^{2}}, \\
\gamma_{x y}^{\mathrm{p}}=-2 z \frac{\partial^{2} w(x, y, t)}{\partial x \partial y},
\end{array}\right.
$$

where $\varepsilon_{x}^{\mathrm{p}}, \varepsilon_{y}^{\mathrm{p}}$, and $\gamma_{x y}^{\mathrm{p}}$ are the strains in the $x, y$, and $x-y$ directions, respectively, and the superscript of ' $p$ ' denotes the piezoelectric patch.

For a piezoelectric patch, its constitutive equations can be reduced to a $2 \mathrm{D}$ form as

$$
\left[\begin{array}{c}
\varepsilon_{x}^{\mathrm{p}} \\
\varepsilon_{y}^{\mathrm{p}} \\
\gamma_{x y}^{\mathrm{p}} \\
D_{3}
\end{array}\right]=\left[\begin{array}{cccc}
s_{11}^{E} & s_{12}^{E} & 0 & d_{31} \\
s_{12}^{E} & s_{11}^{E} & 0 & d_{31} \\
0 & 0 & s_{66}^{E} & 0 \\
d_{31} & d_{31} & 0 & e_{33}^{T}
\end{array}\right]\left[\begin{array}{c}
\sigma_{x}^{\mathrm{p}} \\
\sigma_{y}^{\mathrm{p}} \\
\tau_{x y}^{\mathrm{p}} \\
E_{3}
\end{array}\right],
$$

where $s_{11}^{E}, s_{12}^{E}, s_{66}^{E}$ are elastic compliance parameters at constant electric field $E, d_{31}$ is the piezoelectric constant in the 3-1 direction, $\varepsilon_{33}^{\mathrm{T}}$ is the dielectric permittivity at constant stress $T$, and $D_{3}$ and $E_{3}$ are the electric displacement and the electric field along the $z$-axis, respectively. Furthermore, equation (5) can be rewritten as

$$
\left[\begin{array}{c}
\sigma_{x}^{\mathrm{p}} \\
\sigma_{y}^{\mathrm{p}} \\
\tau_{x y}^{\mathrm{p}} \\
D_{3}
\end{array}\right]=\left[\begin{array}{cccc}
\bar{c}_{11} & \bar{c}_{12} & 0 & -\bar{e}_{31} \\
\bar{c}_{12} & \bar{c}_{11} & 0 & -\bar{e}_{31} \\
0 & 0 & \bar{c}_{66} & 0 \\
\bar{e}_{31} & \bar{e}_{31} & 0 & \bar{\varepsilon}_{33}^{\mathrm{s}}
\end{array}\right]\left[\begin{array}{c}
\varepsilon_{x}^{\mathrm{p}} \\
\varepsilon_{y}^{\mathrm{p}} \\
\gamma_{x y}^{\mathrm{p}} \\
E_{3}
\end{array}\right],
$$

where $\bar{c}_{11}=s_{11}^{E} /\left(\left(s_{11}^{E}\right)^{2}-\left(s_{12}^{E}\right)^{2}\right), \bar{c}_{12}=-\left(s_{11}^{E} /\left(\left(s_{11}^{E}\right)^{2}-\left(s_{12}^{E}\right)^{2}\right)\right)$, $\bar{c}_{66}=1 / s_{66}^{E}, \bar{e}_{31}=d_{31} /\left(s_{11}^{E}+s_{12}^{E}\right)$, and $\bar{\varepsilon}_{33}^{\mathrm{s}}=e_{33}^{T}-\left(2\left(d_{31}\right)^{2} /\left(s_{11}^{E}+\right.\right.$ $\left.\left.s_{12}^{E}\right)\right)$.

By combining equations (4) and (6), the relationship between the stress and transverse displacement of a piezoelectric patch can be obtained as

$$
\left\{\begin{array}{l}
\sigma_{x}^{\mathrm{p}}=-\bar{c}_{11} z \frac{\partial^{2} w(x, y, t)}{\partial x^{2}}-\bar{c}_{12} z \frac{\partial^{2} w(x, y, t)}{\partial y^{2}}-\bar{e}_{31} E_{3} \\
\sigma_{y}^{\mathrm{p}}=-\bar{c}_{12} z \frac{\partial^{2} w(x, y, t)}{\partial x^{2}}-\bar{c}_{11} z \frac{\partial^{2} w(x, y, t)}{\partial y^{2}}-\bar{e}_{31} E_{3} \\
\tau_{x y}^{\mathrm{p}}=-2 \bar{c}_{66} z \frac{\partial^{2} w(x, y, t)}{\partial x \partial y}
\end{array}\right.
$$

2.2.2. Hamilton's Principle-Based Dynamic Model of the $P M P-L R$. In order to characterize vibration propagation behaviors of the PMP-LR, this paper uses Hamilton's principle as equation (8) to derive the dynamic equation of motion for the PMP-LR.

$$
\delta \int_{t_{1}}^{t_{2}}(\mathrm{KE}-\mathrm{PE}) d t=0
$$

where $\delta$ denotes the variational operation and KE and PE are the kinetic and potential energy of the PMP-LR, respectively.

Periodic square holes are cut off from the base structure where piezoelectric patches cover, so the corresponding kinetic and potential energy should not be included. In order to identify the area of the $i^{\text {th }}$ square hole, an indicator function needs to be defined. As shown in Figure 1, the $x$ coordinates of the left and right sides of the $i^{\text {th }}$ piezoelectric patch are denoted as $x_{i, 1}, x_{i, 2}$, respectively. Likewise, the $y$ coordinates of the down and top sides are denoted as $y_{i, 1}, y_{i, 2}$. Then, the indicator function, $\Gamma_{i}(x, y)$, of the $i^{\text {th }}$ piezoelectric patch is defined as

$$
\begin{aligned}
\Gamma_{i}(x, y)= & {\left[\left(H\left(x-x_{i, 1}\right)-H\left(x-x_{i, 2}\right)\right)\right] } \\
& \cdot\left[\left(H\left(y-y_{i, 1}\right)-H\left(y-y_{i, 2}\right)\right)\right],
\end{aligned}
$$

where $H(*)$ is the Heaviside unit step function. Furthermore, the indicator function, $\Gamma(x, y)$, of $N$ piezoelectric patches is defined as

$$
\begin{aligned}
\Gamma(x, y)= & \sum_{i=1}^{N}\left[\left(H\left(x-x_{i, 1}\right)-H\left(x-x_{i, 2}\right)\right)\right] \\
& \cdot\left[\left(H\left(y-y_{i, 1}\right)-H\left(y-y_{i, 2}\right)\right)\right] .
\end{aligned}
$$

Next, the kinetic energy of the PMP-LR (KE) is the sum of the kinetic energies of the base structure $\left(\mathrm{KE}_{\mathrm{S}}\right)$, piezoelectric patches $\left(\mathrm{KE}_{\mathrm{p}}\right)$, and the mass $\left(\mathrm{KE}_{\mathrm{m}}\right)$, i.e.,

$$
\begin{aligned}
K E= & K E_{\mathrm{s}}+K E_{\mathrm{p}}+K E_{\mathrm{m}}, \\
= & \frac{1}{2} \iint\left[\rho_{\mathrm{s}} h_{\mathrm{s}}+\left(\rho_{\mathrm{p}} h_{\mathrm{p}}-\rho_{\mathrm{s}} h_{\mathrm{s}}\right) \Gamma(x, y)\right]\left(\frac{\partial w(x, y, t)}{\partial t}\right)^{2} d x d y \\
& +\frac{1}{2} \iint m_{0} \delta_{\mathrm{D}}\left(x-\frac{x_{i, 1}+x_{i, 2}}{2}\right) \delta_{\mathrm{D}}\left(y-\frac{y_{i, 1}+y_{i, 2}}{2}\right) \\
& \cdot\left(\frac{\partial w(x, y, t)}{\partial t}\right)^{2} d x d y, \\
\equiv & \frac{1}{2} \iint m(x, y) \dot{w}^{2} d x d y,
\end{aligned}
$$


where $\delta_{\mathrm{D}}(*)$ is the Dirac delta functions, $\rho_{\mathrm{s}}$ and $\rho_{\mathrm{p}}$ are the mass densities of the base structure and the piezoelectric patch, respectively, and $m(x, y)$ denotes the equivalent mass of unit area of the PMP-LR which is defined as

$$
\begin{aligned}
m(x, y)= & \rho_{\mathrm{s}} h_{\mathrm{s}}+\left(\rho_{\mathrm{p}} h_{\mathrm{p}}-\rho_{\mathrm{s}} h_{\mathrm{s}}\right) \Gamma(x, y) \\
& +m_{0} \delta_{\mathrm{D}}\left(x-\frac{x_{i, 1}+x_{i, 2}}{2}\right) \delta_{\mathrm{D}}\left(y-\frac{y_{i, 1}+y_{i, 2}}{2}\right) .
\end{aligned}
$$

Likewise, the potential energy of the PMP-LR (PE) is the sum of the potential energies of the base structure $\left(\mathrm{PE}_{\mathrm{s}}\right)$, piezoelectric patches $\left(\mathrm{PE}_{\mathrm{p}}\right)$, and excitation force $\mathrm{PE}_{\mathrm{F}}$, i.e.,

$$
\mathrm{PE}=\mathrm{PE}_{\mathrm{s}}+\mathrm{PE}_{\mathrm{p}}+\mathrm{PE}_{\mathrm{F}} \text {. }
$$

Furthermore, we will have

$$
\begin{aligned}
\mathrm{PE}_{\mathrm{s}}+\mathrm{PE}_{\mathrm{p}}= & \frac{1}{2} \iiint\left[(1-\Gamma(x, y)) \boldsymbol{\sigma}_{\mathrm{s}}^{\mathrm{T}} \boldsymbol{\varepsilon}_{\mathrm{s}}+\Gamma(x, y) \mathbf{\sigma}_{\mathrm{p}}^{\mathrm{T}} \boldsymbol{\varepsilon}_{\mathrm{p}}\right] d x d y d z \\
= & \frac{1}{2} \iiint\left[(1-\Gamma(x, y)) z^{2} \boldsymbol{\Theta}^{\mathrm{T}} \mathbf{c}_{\mathrm{s}}^{E} \boldsymbol{\Theta} w^{2}+\Gamma(x, y)\right. \\
& \left.\cdot\left(z^{2} \boldsymbol{\Theta}^{\mathrm{T}} \mathbf{c}_{\mathrm{p}}^{E} \boldsymbol{\Theta} w^{2}+z \mathbf{e} E_{3} \boldsymbol{\Theta}^{\mathrm{T}} w\right)\right] d x d y d z \\
= & \frac{h^{3}}{24} \iint\left[\boldsymbol{\Theta}^{\mathrm{T}}\left((1-\Gamma(x, y)) \mathbf{c}_{\mathrm{s}}^{E}+\Gamma(x, y) \mathbf{c}_{\mathrm{p}}^{E}\right) \boldsymbol{\Theta} w^{2}\right] d x d y \\
& -\frac{h_{\mathrm{p}}-h}{4} \iint \Gamma(x, y)\left(\mathbf{e} V(t) \boldsymbol{\Theta}^{\mathrm{T}} w\right) d x d y,
\end{aligned}
$$

where $E_{3}=-V(t) / h_{\mathrm{p}}, \Theta=\left[\begin{array}{lll}\partial^{2} / \partial^{2} x & \partial^{2} / \partial^{2} y & 2 \partial^{2} / \partial x \partial y\end{array}\right]^{\mathrm{T}}$, $\boldsymbol{\sigma}_{s}=\left[\sigma_{x}^{s}, \sigma_{y}^{s}, \tau_{x y}^{s}\right]^{\mathrm{T}}, \boldsymbol{\sigma}_{p}=\left[\sigma_{x}^{p}, \sigma_{y}^{p}, \tau_{x y}^{p}\right]^{\mathrm{T}}, \boldsymbol{\varepsilon}_{s}=\left[\begin{array}{lll}\varepsilon_{x}^{s} & \varepsilon_{y}^{s} & \gamma_{x y}^{s}\end{array}\right]^{\mathrm{T}}$, $\boldsymbol{\varepsilon}_{p}=\left[\begin{array}{lll}\varepsilon_{x}^{p} & \varepsilon_{y}^{p} & \gamma_{x y}^{p}\end{array}\right]^{\mathrm{T}}, \mathbf{c}_{\mathrm{p}}^{E}=1 /\left(s_{11}^{E}\left(1-\mu_{\mathrm{p}}^{2}\right)\right)\left[\begin{array}{ccc}1 & \mu_{\mathrm{p}} & 0 \\ \mu_{\mathrm{p}} & 1 & 0 \\ 0 & 0 & \left(1-\mu_{\mathrm{p}}\right) / 2\end{array}\right]$, $\mathbf{c}_{\mathrm{s}}^{E}=E_{\mathrm{s}} /\left(1-\mu_{\mathrm{s}}^{2}\right)\left[\begin{array}{ccc}1 & \mu_{\mathrm{s}} & 0 \\ \mu_{\mathrm{s}} & 1 & 0 \\ 0 & 0 & \left(1-\mu_{\mathrm{s}}\right) / 2\end{array}\right], \quad$ and $\quad \mathbf{e}=d_{31} /\left(s_{11}^{E}(1-\right.$ $\left.\left.\mu_{\mathrm{p}}\right)\right)\left[\begin{array}{l}1 \\ 1 \\ 0\end{array}\right]$, and

$$
\mathrm{PE}_{\mathrm{F}}=-\iint F\left(x_{0}, y_{0}, t\right) w d x d y .
$$

Finally, each term in the left side of equation (8) can be calculated as equations (16) (19).

$$
\begin{aligned}
\int_{t_{1}}^{t_{2}} \delta \mathrm{KE} d t & =\int_{t_{1}}^{t_{2}} \delta\left[\frac{1}{2} \iint m(x, y) \dot{w}^{2} d x d y\right] d t \\
& =-\int_{t_{1}}^{t_{2}} \iint m(x, y) \ddot{w} \delta w d x d y d t,
\end{aligned}
$$

$$
\begin{aligned}
& \int_{t_{1}}^{t_{2}} \delta\left\{\frac{h^{3}}{24} \iint\left[\boldsymbol{\Theta}^{\mathrm{T}}\left((1-\Gamma(x, y)) \mathbf{c}_{\mathrm{s}}^{E}+\Gamma(x, y) \mathbf{c}_{\mathrm{p}}^{E}\right) \boldsymbol{\Theta} w^{2}\right] d x d y\right\} d t \\
&=\int_{t_{1}}^{t_{2}} \iint \boldsymbol{\Theta}^{\mathrm{T}} \mathbf{C} \boldsymbol{\Theta} w \delta w d x d y d t,
\end{aligned}
$$

where $\mathbf{C}=\left(h^{3} / 12\right)\left[(1-\Gamma(x, y)) \mathbf{c}_{\mathrm{s}}^{E}+\Gamma(x, y) \mathbf{c}_{\mathrm{p}}^{E}\right]$.

$$
\begin{array}{r}
\int_{t_{1}}^{t_{2}} \delta\left[-\frac{h_{\mathrm{p}}-h}{4} \iint \Gamma(x, y)\left(\mathbf{e} V(t) \Theta^{\mathrm{T}} w\right) d x d y\right] d t \\
=\frac{h-h_{\mathrm{p}}}{4} \int_{t_{1}}^{t_{2}} \iint \Gamma(x, y) \mathbf{e}^{\mathrm{T}} V(t) \delta w d x d y d t, \\
\delta \int_{t_{1}}^{t_{2}} \mathrm{PE}_{\mathrm{F}} d t=-\delta \int_{t_{1}}^{t_{2}} \iint F\left(x_{0}, y_{0}, t\right) w d x d y d t \\
=-\int_{t_{1}}^{t_{2}} \iint F\left(x_{0}, y_{0}, t\right) \delta w d x d y d t .
\end{array}
$$

Substituting equations (16) (19) into equation (8), we can obtain the electromechanically coupled vibration propagation model of the PMP-LR as follows by neglecting the damping term:

$$
\begin{aligned}
& m(x, y) \frac{\partial^{2} w(x, y, t)}{\partial t^{2}}+\Theta^{T}\left[\mathbf{C}(\Theta w)+\frac{h-h_{\mathrm{p}}}{4} \Gamma(x, y) \mathbf{e} V(t)\right] \\
& =F\left(x_{0}, y_{0}, t\right) .
\end{aligned}
$$

Compared with traditional thin plates, it can be seen that a voltage term due to piezoelectric effect is added into equation (20).

2.3. Modal Analysis of the PMP-LR Based on the Rayleigh-Ritz Method. In order to explore vibration characteristics of the PMP-LR, modal analysis is one common way which can be used to obtain natural frequencies and corresponding mode shapes under a given boundary condition. Obviously, it is difficult to obtain an exact solution of the PMP-LR according to equation (20). Similar to existing works $[8,10]$, the Rayleigh-Ritz method will be implemented to perform modal vibration analysis in this paper.

Based on the Rayleigh-Ritz method, vibration displacements of the PMP-LR can be written as equation (21) by using the assuming mode:

$$
w(x, y, t)=\sum_{m=1}^{N} \sum_{n=1}^{N} \phi_{m n}(x, y) \varphi_{m n}(t),
$$

where $m n$ denotes the $(m, n)$ order, $\phi_{m n}(x, y)$ is the $(m, n)$-order assumed mode shape, and $\varphi_{m n}(t)$ represents the modal coordinates.

Furthermore, $\phi_{m, n}(x, y)$ can be rewritten as

$$
\phi_{m, n}(x, y)=\xi_{m}(x) \eta_{n}(y),
$$

where $\xi_{m}(x)$ and $\eta_{n}(y)$ are the trial functions in the $x$ and $y$ directions, respectively.

For the PMP-LR in Figure 2, three edges are clamped and one edge is fixed. These conditions are called Clamped-FreeFree-Free (CFFF) boundary conditions. That is to say, it can be considered a cantilever beam in the $x$ direction, while a free beam in the $y$ direction. Then, the trial functions in the $x$ and $y$ directions can be represented as follows: 


$$
\begin{aligned}
\xi_{m}(x)= & \operatorname{ch} \frac{\lambda_{m}}{a} x-\cos \frac{\lambda_{m}}{a} x \\
& -\frac{\left(\operatorname{sh} \lambda_{m}-\sin \lambda_{m}\right)\left(\operatorname{sh}\left(\lambda_{m} / a\right) x-\sin \left(\lambda_{m} / a\right) x\right)}{\left(\operatorname{ch} \lambda_{m}+\cos \lambda_{m}\right)}
\end{aligned}
$$

where $\lambda_{1}=1.571, \lambda_{2}=4.694, \lambda_{3}=7.855$, and $\lambda_{m}=(m-$ $0.5) \pi, \quad m \geq 4$.

$$
\begin{aligned}
\eta_{n}(y)= & \operatorname{ch} \frac{\zeta_{n}}{b} y+\cos \frac{\zeta_{n}}{b} y \\
& -\frac{\left(\operatorname{sh} \zeta_{n}+\sin \zeta_{n}\right)\left(\operatorname{sh}\left(\zeta_{n} / b\right) y+\sin \left(\zeta_{n} / b\right) y\right)}{\operatorname{ch} \zeta_{n}-\cos \zeta_{n}}
\end{aligned}
$$

where $\zeta_{1}=4.730, \zeta_{2}=7.853, \zeta_{3}=10.996$, and $\zeta_{m}=(m+$ 0.5) $\pi, \quad m \geq 4$.

Under the open-circuit condition, the effect of $V(t)$ can be neglected. Then, equation (20) will be simplified as equation (25) for the modal analysis.

$$
m(x, y) \frac{\partial^{2} w}{\partial t^{2}}+\Theta^{\mathrm{T}} \mathbf{C}(\Theta w)=0
$$

Substituting equations (21) (24) into equation (25) and multiplying $\phi_{r s}(x, y)$ at both sides, the integration over the whole plate will lead to

$$
-w^{2} M_{r s} \varphi+K_{r s} \varphi=0, \quad r, s=1,2, \ldots, N,
$$

where

$$
\begin{aligned}
M_{r s} & =\int_{0}^{L_{\mathrm{a}}} \int_{0}^{L_{\mathrm{b}}} \phi_{r s}(x, y) m(x, y) \sum_{m=1}^{N} \sum_{n=1}^{N} \phi_{m n}(x, y) d x d y \\
& = \begin{cases}0, & r \neq m \text { or } s \neq n, \\
\int_{0}^{L_{\mathrm{a}}} \int_{0}^{L_{\mathrm{b}}} \phi_{r s}(x, y) m(x, y) \phi_{r s}(x, y) d x d y, & r=m \text { and } s=n,\end{cases} \\
K_{r s} & =\int_{0}^{L_{\mathrm{a}}} \int_{0}^{L_{\mathrm{b}}} \phi_{r s}(x, y) \Theta^{\mathrm{T}} \mathbf{C} \Theta \sum_{m=1}^{N} \sum_{n=1}^{N} \phi_{m n}(x, y) d x d y \\
& = \begin{cases}0, & r \neq m \text { or } s \neq n, \\
\int_{0}^{L_{\mathrm{a}}} \int_{0}^{L_{\mathrm{b}}}\left[\Theta \phi_{r s}(x, y)\right]^{\mathrm{T}} \mathbf{C} \Theta \phi_{r s}(x, y) d x d y, & r=m \text { and } s=n .\end{cases}
\end{aligned}
$$

Furthermore, the matrix form of equation (26) can be written as

$$
\left(\omega^{2} \mathbf{M}-\mathbf{K}\right)\{\boldsymbol{\varphi}\}=0
$$

Obviously, equation (27) is just the eigenvalue equation of the undamped PMP-LR. Based on equation (27), the eigenfrequency $\left(\omega_{j}\right)$ and the corresponding eigenvector $\left(\{\boldsymbol{\varphi}\}^{j}, \quad j=1,2, \ldots, N\right)$ can be calculated. Finally, the vibration shape corresponding to $\omega_{j}$ can be obtained as $\phi_{j j}(x, y)\{\boldsymbol{\varphi}\}^{j}$.

\section{Vibration Bandgaps of the PMP-LR Based on the Bloch Theorem}

In order to estimate vibration bandgaps, the PMP-LR is assumed to be infinitely periodic. In this case, the Bloch theorem can be used to calculate bandgaps [17]. Furthermore, due to the periodicity of the structure, it is only necessary to study one unit cell shown in Figure 1. Then, vibration equation of the unit cell can be discretized as follows by the finite element method:

$$
\left(\mathbf{K}_{\mathrm{c}}-\omega^{2} \mathbf{M}_{\mathrm{c}}\right) \mathbf{u}_{\mathrm{c}}=\mathbf{F}_{\mathrm{c}},
$$

where $\mathbf{K}_{\mathrm{c}}$ and $\mathbf{M}_{\mathrm{c}}$ are the stiffness and mass matrices of the unit cell, respectively, $\mathbf{u}_{c}$ and $\mathbf{F}_{c}$ are the generalized displacements and force vectors of the unit cell, respectively, and $\omega$ is the angular frequency of harmonic vibrations. Components of $\mathbf{u}_{\mathrm{c}}$ and $\mathbf{F}_{\mathrm{c}}$ can be represented as Figure 3 .

Based on Figure 3, we will have

$$
\begin{aligned}
& \mathbf{u}_{\mathrm{c}}=\left[\begin{array}{llllllll}
\mathbf{u}_{\mathrm{B}}^{\mathrm{T}} & \mathbf{u}_{\mathrm{T}}^{\mathrm{T}} & \mathbf{u}_{\mathrm{LB}}^{\mathrm{T}} & \mathbf{u}_{\mathrm{LT}}^{\mathrm{T}} & \mathbf{u}_{\mathrm{RB}}^{\mathrm{T}} & \mathbf{u}_{\mathrm{RT}}^{\mathrm{T}} & \mathbf{u}_{\mathrm{L}}^{\mathrm{T}} & \mathbf{u}_{\mathrm{R}}^{\mathrm{T}}
\end{array}\right]^{\mathrm{T}}, \\
& \mathbf{F}_{\mathrm{c}}=\left[\begin{array}{llllllll}
\mathbf{F}_{\mathrm{B}}^{\mathrm{T}} & \mathbf{F}_{\mathrm{T}}^{\mathrm{T}} & \mathbf{F}_{\mathrm{LB}}^{\mathrm{T}} & \mathbf{F}_{\mathrm{LT}}^{\mathrm{T}} & \mathbf{F}_{\mathrm{RB}}^{\mathrm{T}} & \mathbf{F}_{\mathrm{RT}}^{\mathrm{T}} & \mathbf{F}_{\mathrm{L}}^{\mathrm{T}} & \mathbf{F}_{\mathrm{R}}^{\mathrm{T}}
\end{array}\right]^{\mathrm{T}},
\end{aligned}
$$

where the subscripts $\mathrm{L}, \mathrm{R}, \mathrm{B}$, and T denote four boundaries (left, right, bottom, and top) of the unit cell and LB, RB, LT, and RT denote its four corners (left-bottom, right-bottom, left-top, and right-top). Based on the Bloch theorem, displacements vectors on the cell's boundaries will satisfy

$$
\begin{aligned}
& \mathbf{u}_{\mathrm{R}}^{\mathrm{T}}=e^{-i k_{x} a_{x}} \mathbf{u}_{\mathrm{L}}^{\mathrm{T}}, \\
& \mathbf{u}_{\mathrm{T}}^{\mathrm{T}}=e^{-i k_{x} a_{x}} \mathbf{u}_{\mathrm{B}}^{\mathrm{T}} .
\end{aligned}
$$

Next, displacement vectors on the cell's corners will satisfy equations (32) (36).

$$
\begin{aligned}
& \mathbf{u}_{\mathrm{RB}}^{\mathrm{T}}=e^{-i k_{x} a_{x}} \mathbf{u}_{\mathrm{LB}}^{\mathrm{T}}, \\
& \mathbf{u}_{\mathrm{LT}}^{\mathrm{T}}=e^{-i k_{x} a_{y}} \mathbf{u}_{\mathrm{LB}}^{\mathrm{T}}, \\
& \mathbf{u}_{\mathrm{RT}}^{\mathrm{T}}=e^{-i k_{x} a_{x}-i k_{y} a_{y}} \mathbf{u}_{\mathrm{LB}}^{\mathrm{T}},
\end{aligned}
$$




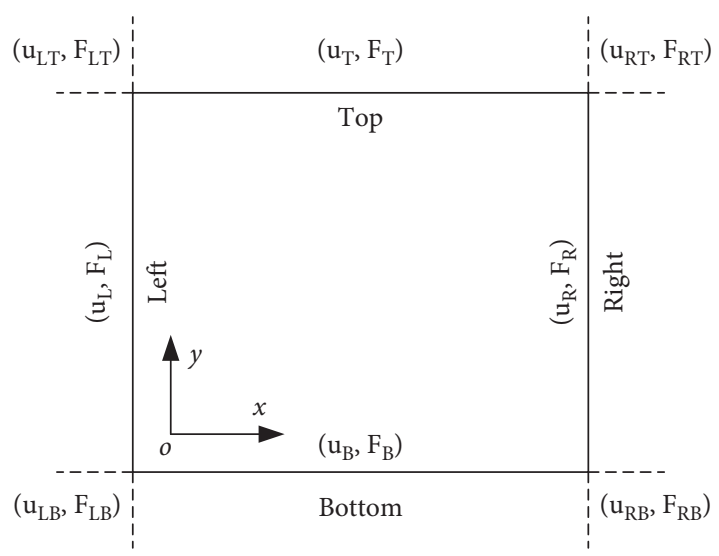

FIGURE 3: Schematic diagram of components of displacement and forces.

where $k_{x}$ and $k_{y}$ denote components of wave vector $(k)$ in the $x$ and $y$ directions, respectively, and $a_{x}$ and $a_{y}$ represent lattice constants in the $x$ and $y$ directions. Then, equations (29) (32) can be written as the following matrix:

$$
\begin{aligned}
\mathbf{u}_{\mathrm{c}} & =\left[\begin{array}{c}
\mathbf{u}_{\mathrm{B}} \\
\mathbf{u}_{\mathrm{T}} \\
\mathbf{u}_{\mathrm{LB}} \\
\mathbf{u}_{\mathrm{LT}} \\
\mathbf{u}_{\mathrm{RB}} \\
\mathbf{u}_{\mathrm{RT}} \\
\mathbf{u}_{\mathrm{L}} \\
\mathbf{u}_{\mathrm{R}}
\end{array}\right]=\left[\begin{array}{ccc}
\mathbf{I}_{\mathrm{B}} & 0 & 0 \\
e^{-i k_{y} a_{y}} \mathbf{I}_{\mathrm{T}} & 0 & 0 \\
0 & \mathbf{I}_{\mathrm{LB}} & 0 \\
0 & e^{-i k_{y} a_{y}} \mathbf{I}_{\mathrm{LT}} & 0 \\
0 & e^{-i k_{x} a_{x}} \mathbf{I}_{\mathrm{RB}} & 0 \\
0 & e^{-i k_{x} a_{x}-i k_{y} a_{y}} \mathbf{I}_{\mathrm{RT}} & 0 \\
0 & 0 & \mathbf{I}_{\mathrm{L}} \\
0 & 0 & e^{-i k_{y} a_{y}} \mathbf{I}_{\mathrm{R}}
\end{array}\right]\left[\begin{array}{c}
\mathbf{u}_{\mathrm{B}} \\
\mathbf{u}_{\mathrm{LB}} \\
\mathbf{u}_{\mathrm{L}}
\end{array}\right] \\
& =\mathbf{T}\left(k_{x}, k_{y}\right) \mathbf{u}_{\mathrm{c}}^{\prime},
\end{aligned}
$$

where I denotes the identity matrix.

Similarly, the force vectors on the cell's boundaries can be derived by the Bloch theorem. Boundary forces of four adjacent unit cells are shown in Figure 4, and the equilibrium force conditions can be expressed as equations (35) (37).

$$
\begin{array}{r}
\mathbf{F}_{\mathrm{R}}+e^{-i k_{x} a_{x}} \mathbf{F}_{\mathrm{L}}=0, \\
\mathbf{F}_{\mathrm{T}}+e^{-i k_{y} a_{y}} \mathbf{F}_{\mathrm{B}}=0, \\
\mathbf{F}_{\mathrm{RT}}+e^{-i k_{x} a_{x}} \mathbf{F}_{\mathrm{LT}}+e^{-i k_{y} a_{y}} \mathbf{F}_{\mathrm{RB}}+e^{-i k_{x} a_{x}-i k_{y} a_{y}} \mathbf{F}_{\mathrm{LB}}=0 .
\end{array}
$$

Substituting equation (36) into equation (28), we will have

$$
\left(\mathbf{K}_{\mathrm{c}}-\omega^{2} \mathbf{M}_{\mathrm{c}}\right) \mathbf{T}\left(k_{x}, k_{y}\right) \mathbf{u}_{\mathrm{c}}^{\prime}=\mathbf{F}_{\mathrm{c}} .
$$

Multiplying $\mathbf{T}^{\mathrm{H}}\left(k_{x}, k_{y}\right)$ with both sides of (39), we will have

$$
\left[\mathbf{K}_{\mathrm{c}}^{\prime}\left(k_{x}, k_{y}\right)-\omega^{2} \mathbf{M}_{\mathrm{c}}^{\prime}\left(k_{x}, k_{y}\right)\right] \mathbf{u}_{\mathrm{c}}^{\prime}=0
$$

where

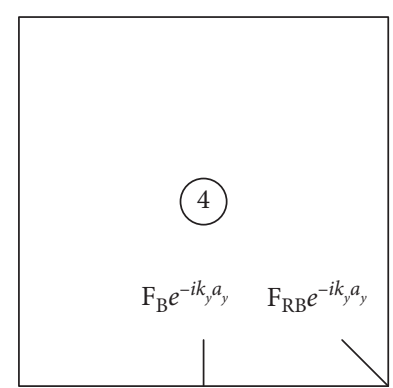

(a)

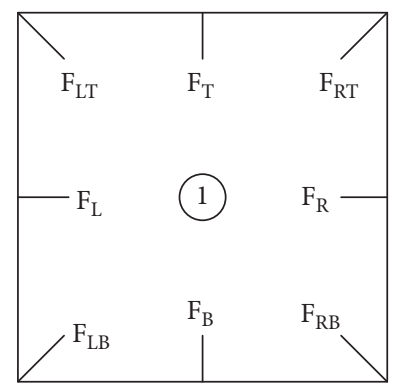

(c)

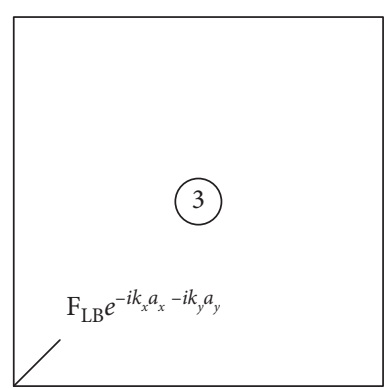

(b)

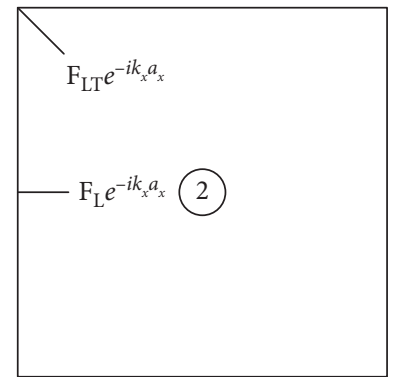

(d)
Figure 4: Boundary forces of four adjacent unit cells.

$$
\begin{aligned}
& \mathbf{K}_{\mathrm{c}}^{\prime}\left(k_{x}, k_{y}\right)=\mathbf{T}^{\mathrm{H}}\left(k_{x}, k_{y}\right) \mathbf{K}_{\mathrm{c}} \mathbf{T}\left(k_{x}, k_{y}\right), \\
& \mathbf{M}_{\mathrm{c}}^{\prime}\left(k_{x}, k_{y}\right)=\mathbf{T}^{\mathrm{H}}\left(k_{x}, k_{y}\right) \mathbf{M}_{\mathrm{c}} \mathbf{T}\left(k_{x}, k_{y}\right),
\end{aligned}
$$

represent reduced stiffness and mass matrices, respectively.

Based on equation (41), we can obtain the corresponding value of $\omega$ once the wave vector $\left(k_{x}, k_{y}\right)$ is specified. Generally, the wave vector is always chosen along the edges of the irreducible part of the first Brillouin zone. In this case, the dispersion curve can be calculated, where vibration bandgaps can be obtained. Main direction (MGX) in the reciprocal space is shown as $\overrightarrow{\Gamma M}$ in Figure 5, where vibration modal frequencies can be obtained from the dispersion curve.

\section{Finite Element Method-Based Vibration Simulations}

For vibration energy harvesting in practice, there is a need to design optimally the PMP-LR to meet the demands of lowfrequency and broad bandwidths. Therefore, we will study vibration characteristics of the PMP-LR by using the proposed method based on finite element analysis. Firstly, the COMSOL Multiphysics 5.2a software is used to build the entity model of the unit cell, and material properties in simulations are listed in Table 1 . Then, periodic boundary conditions as shown in Section 3 are set, and the finite element model is built. Finally, simulations are done and the corresponding results are collected and analyzed.

4.1. Effects of Geometric Configurations on Vibration Bandgaps of the PMP-LR. For the local resonator, there are possible geometric configurations shown in Figure 6 


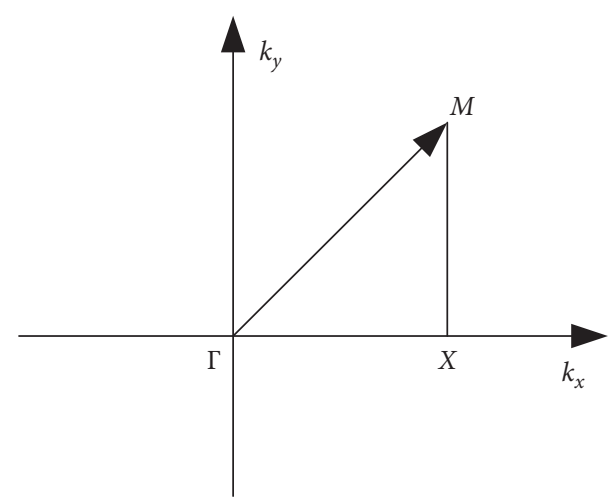

Figure 5: Main direction (MGX) in the reciprocal space.

TABLE 1: Material properties of the PMP-LR in simulations.

\begin{tabular}{lcccc}
\hline & Material & $\begin{array}{c}\text { Young's } \\
\text { modulus } E(\mathrm{GPa})\end{array}$ & $\begin{array}{c}\text { Poisson's } \\
\text { ratio }(v)\end{array}$ & $\begin{array}{c}\text { Density } \\
\rho\left(\mathrm{kg} / \mathrm{m}^{3}\right)\end{array}$ \\
\hline PZT base & $\mathrm{Al}$ & 70 & 0.33 & 2700 \\
PZT patch & PZT-5H & 31 & 0.29 & 7500 \\
Mass & Steel & 200 & 0.30 & 7870 \\
\hline
\end{tabular}

according to the relative positions of piezoelectric layer, elastic base, and mass. Compared with the host plate and the mass, the piezoelectric layer is almost sandwiched under type 1 and type 3 , which is similar to reference [18]. We will investigate the locations and widths of bandgaps under four different types. Geometric dimensions of the unit cell in simulations are listed in Table 2, and the lattice constant is equal to $100 \mathrm{~mm}$. By using the proposed method in Section 3 , The dispersion curves under type 1 , type 2 , type 3 , and type 4 are calculated and shown in Figures 7 10, respectively.

In Figures 7 10, the signs of " $\bigcirc$," " $\Delta$," and " $\square$ " denote the first, second, and third modal frequencies, respectively, according to Figure 5. Obviously, the second and third modal frequencies are very close. Particularly, the shaded area denotes the corresponding bandgap. Based on these results, we can obtain its upper bound, lower bound, and width under four geometric configurations, as shown in Table 3 .

It can be seen from Table 3 that geometric configuration of the local resonator has some effects on the location of bandgap, while the width hardly changes. Among the four types, the frequency location of bandgap is the lowest, and its width is the largest under type 3.

In order to validate the locally resonant bandgap, the corresponding wavelength $\lambda$ can be estimated as $\lambda=v /$ $f_{\text {bandgap }}\left(v\right.$ is the transverse wave velocity and $f_{\text {bandgap }}$ is the bandgap width). Furthermore, $v$ can be calculated as $v=$ $\sqrt{E_{\mathrm{s}}\left(1+\mu_{\mathrm{s}}\right) / 2 \rho_{\mathrm{s}}}\left(\rho_{\mathrm{s}}\right.$ are the density of the structural layer). Taking type 3 as an example, $f_{\text {bandgap }}=106 \mathrm{~Hz}$ and we can obtain $\lambda=41.52 \mathrm{~m}$. The lattice constant is $100 \mathrm{~mm}$, so the reduced size ratio is about 0.00235 (eg., $0.1 / 42.52$ ). Thus, we can conclude that the bandgap is not caused by Bragg scattering.

4.2. Effects of Geometric Parameters on Vibration Bandgaps of the PMP-LR. Under type 3, we will explore effects of geometric parameters of the local resonator on vibration bandgaps. Here the lattice constant is equal to $40 \mathrm{~mm}$. Firstly, the length of PZT-5H changes from $15.5 \mathrm{~mm}$ to $37.9 \mathrm{~mm}$, and the corresponding lower bound, upper bound, and width of the bandgap are calculated and plotted in Figures 11 and 12.

It can be seen from Figures 11 and 12 that both the lower bound and the upper bound decrease with the length of PZT-5H, while the bandwidth first decreases and then increases with the length of PZT-5H.

Next, the thickness of PZT-5H will affect the bandgap due to two theoretic reasons. The first one is that the bending stiffness is directly proportional to the cube of thickness. The other one is that the thickness is strongly related to the parameters in piezoelectric equations. Thus, the thickness of PZT-5H is changed, and the corresponding lower bound, upper bound, and width of the bandgap are calculated and plotted in Figures 13 and 14, where we can see that both bounds increase with the increase in thickness, while the bandwidth first increases and then decreases with the thickness of PZT-5H.

In the end, we change the square mass, and the corresponding lower bound, upper bound, and width of the bandgap are calculated and plotted in Figures 15 and 16, where we can see that both the lower bound and the upper bound decrease with the square mass, while the bandwidth increases with the square mass.

Based on Figures 11 16, we can conclude that both increasing the length of PZT-5H and decreasing the thickness of PZT-5H can make the vibration bandgap shift to low-frequency regions under a given lattice constant. At the same time, there exist optimal length and thickness of PZT-5H to obtain the maximum bandwidth. In addition, increasing the square mass can make the vibration bandgap shift to low-frequency regions and increase the bandwidth. Therefore, for low-frequency and broadband vibration energy harvesting in engineering applications, we should select optimal length and thickness of PZT-5H and large mass to build the local resonator based on concrete requirements. Similar conclusions can be obtained for the other three types according to simulated results which are not shown due to the limitation of length.

4.3. Vibration Modals of the PMP-LR. In this section, we will use the method proposed in Section 2.3 to calculate vibration modals of a PMP-LR. Here a PMP-LR composed of $4 \times 4$ unit cells is considered, and wave vectors are selected as $k_{x}=k_{y}=1$. Then, the first six vibration mode shapes are calculated, and the displacement field is shown in Figure 17. It can be found from Figure 17 that vibration localizations appear in the third, fourth, fifth, and sixth vibration mode shapes. That is to say, vibration energy just concentrates on some unit cells, which means that these local resonators are forced to be resonant as expected.

Furthermore, piezoelectric voltages under the first six modes are shown in Figure 18. 


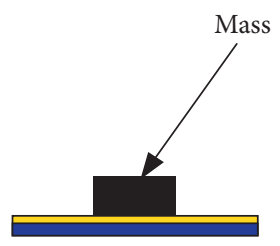

(a)

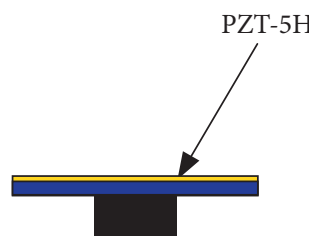

(b)

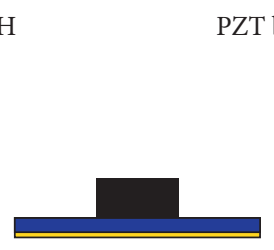

(c)

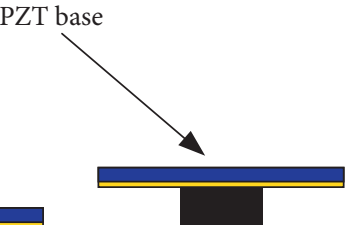

(d)

Figure 6: Four candidate configurations of the local resonator. (a) Type 1. (b) Type 2. (c) Type 3. (d) Type 4.

TABLE 2: Geometric dimensions of the unit cell in simulations.

\begin{tabular}{lcccc}
\hline & PZT $(\mathrm{mm})$ & PZT base $(\mathrm{mm})$ & Mass $(\mathrm{mm})$ & \\
\hline Length & 90 & 90 & 30 & Plate $(\mathrm{mm})$ \\
Width & 90 & 90 & 30 & 100 \\
Thickness & 0.2 & 0.5 & 15 & 100 \\
\hline
\end{tabular}

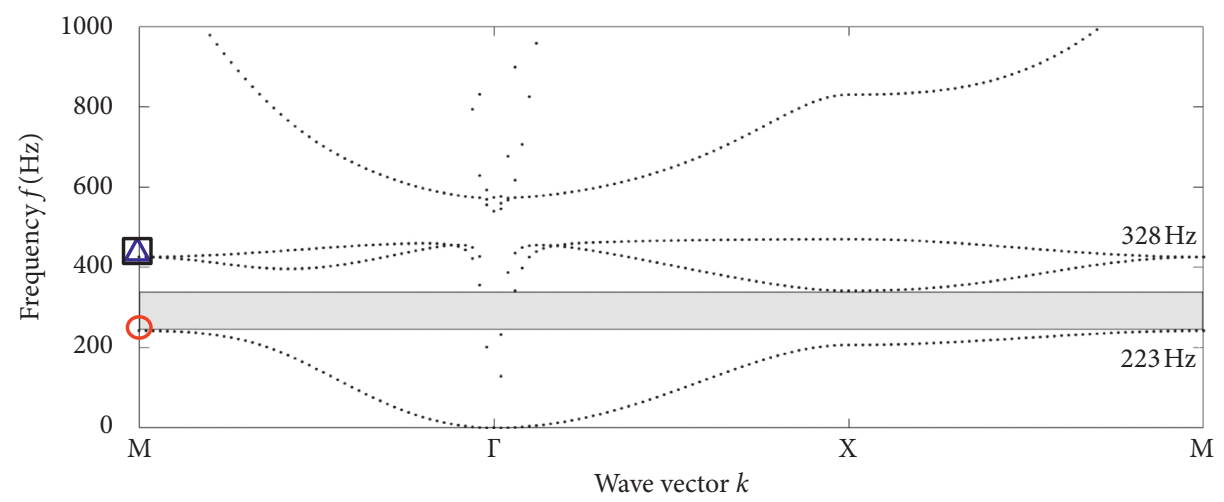

Figure 7: Dispersion curve under type 1.

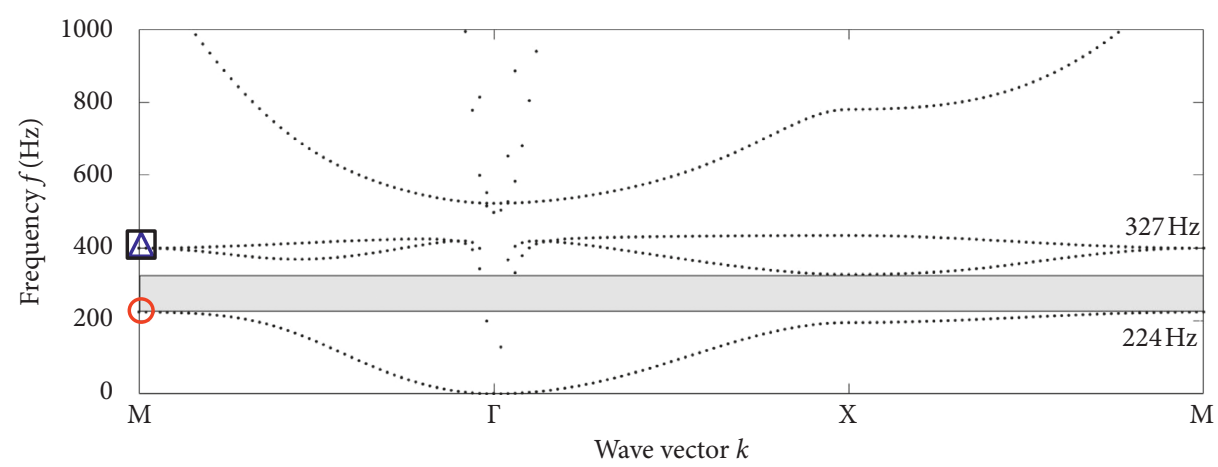

FIGURE 8: Dispersion curve under type 2.

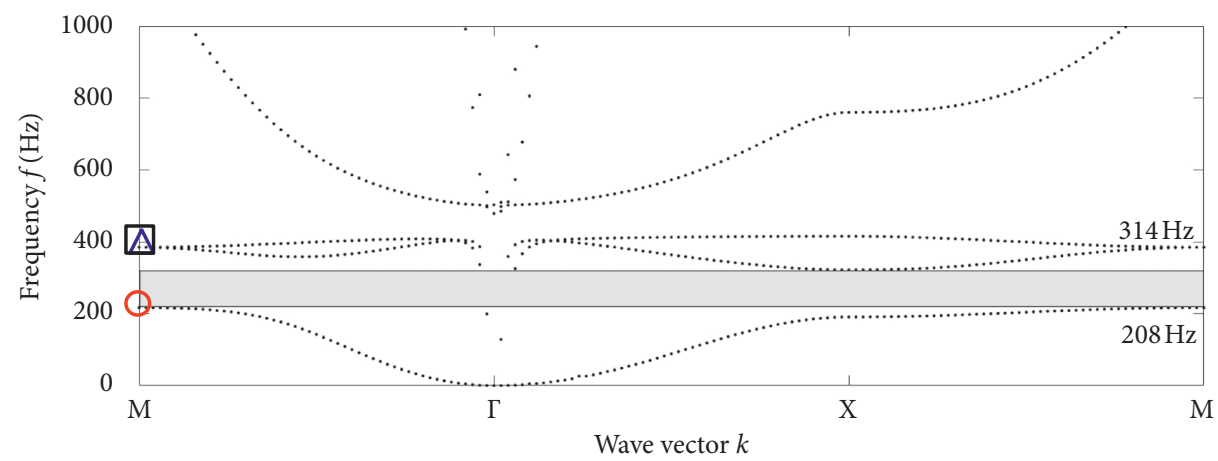

FIGURE 9: Dispersion curve under type 3. 


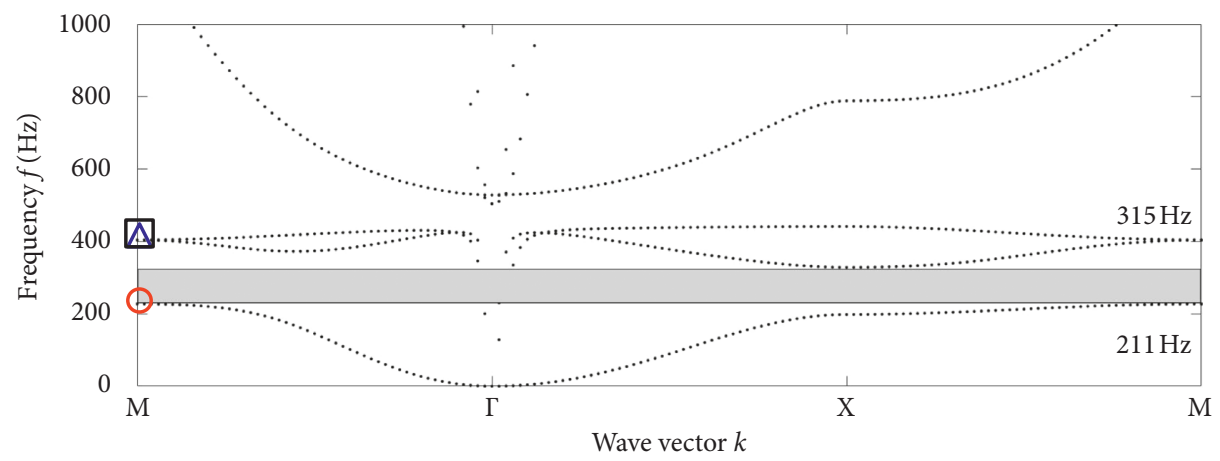

FIgURE 10: Dispersion curve under type 4.

TABlE 3: Comparison of bandgaps under four geometric configurations.

\begin{tabular}{lcccc}
\hline Bandgap & Type 1 & Type 2 & Type 3 & Type 4 \\
\hline Lower bound & $223 \mathrm{~Hz}$ & $224 \mathrm{~Hz}$ & $208 \mathrm{~Hz}$ & $211 \mathrm{~Hz}$ \\
Upper bound & $328 \mathrm{~Hz}$ & $327 \mathrm{~Hz}$ & $314 \mathrm{~Hz}$ & $315 \mathrm{~Hz}$ \\
Width & $105 \mathrm{~Hz}$ & $103 \mathrm{~Hz}$ & $106 \mathrm{~Hz}$ & $104 \mathrm{~Hz}$ \\
\hline
\end{tabular}

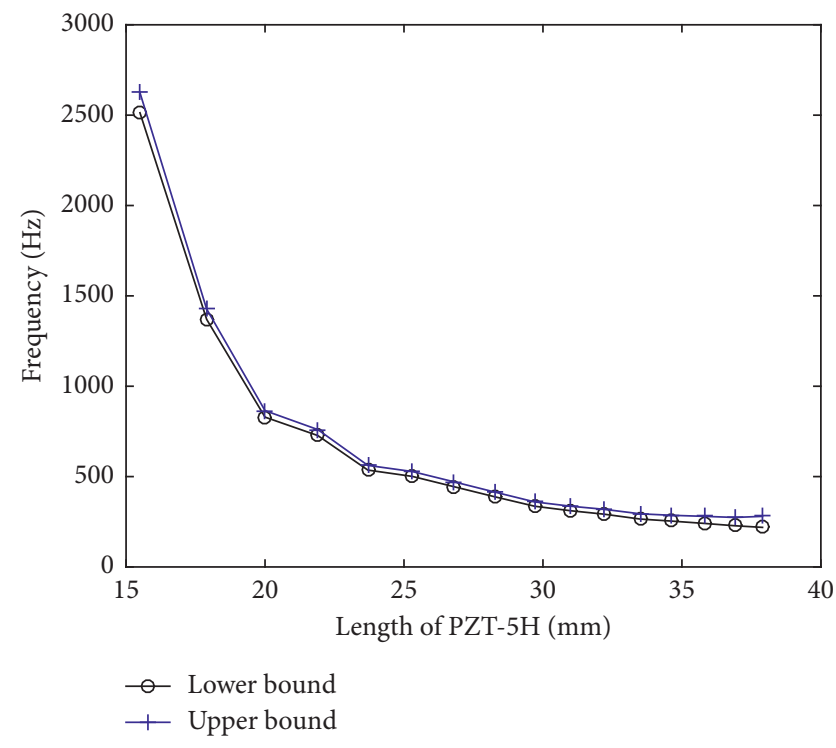

FIgUre 11: The lower and upper bound vs. the length of PZT-5H.

\section{Experimental Validations}

In order to validate theoretic and simulated results, an experimental setup is built as shown in Figure 19, which is composed of a Polytec vibration scanner, a signal sampling system, a power amplifier, a vibration shaker, and a PMPLR. Then, a PMP-LR composed of $4 \times 4$ unit cells is fabricated as shown in Figure 19(b), and its geometric and material parameters are chosen as shown in Tables 1 and 2 . The configuration of the local resonator is type 3 .

The locations of vibration excitations and responses are shown in Figure 19(b). In the experiments, the excitation frequency is swept from $0 \mathrm{~Hz}$ to $1000 \mathrm{~Hz}$. At the same time, the excitation and three response signals are collected and

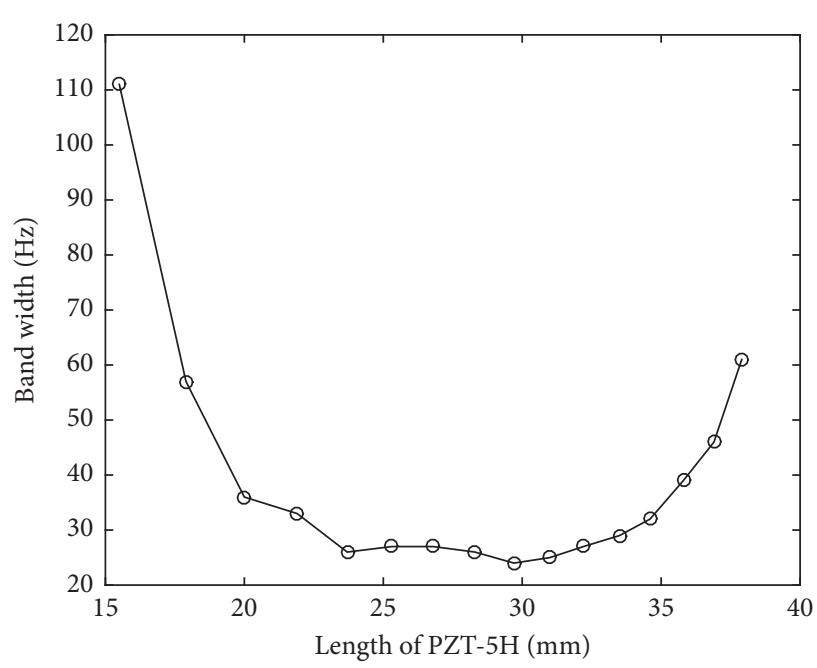

Figure 12: The bandwidth vs. the length of PZT-5H.

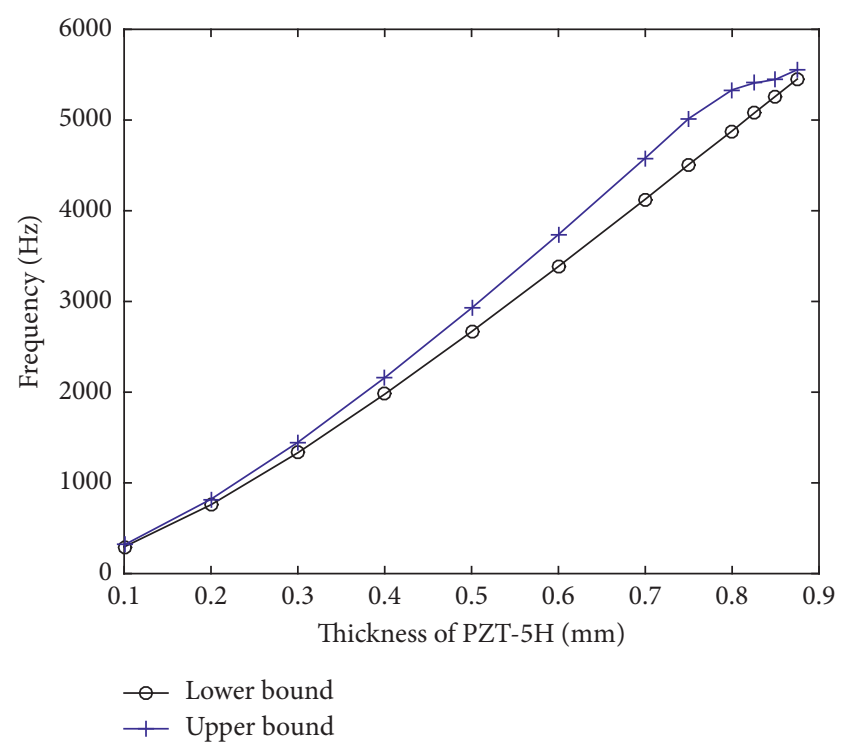

FIGURE 13: Lower and upper bounds vs. thickness of PZT-5H.

their power spectrums are calculated, respectively. Then, the ratio of power spectrums of response signals and excitation signals is defined as vibration propagation function. In the 


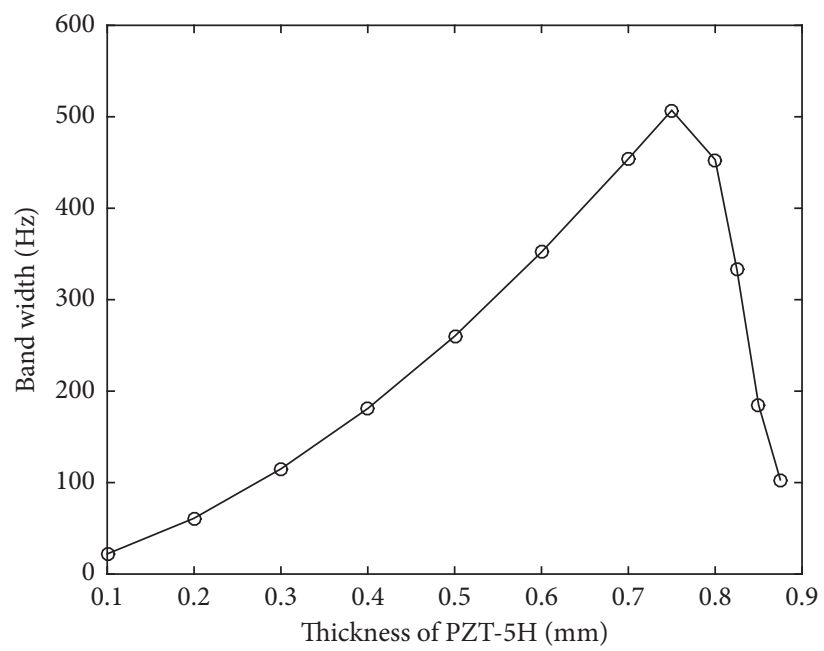

FIGURe 14: Bandwidth vs. thickness of PZT-5H.

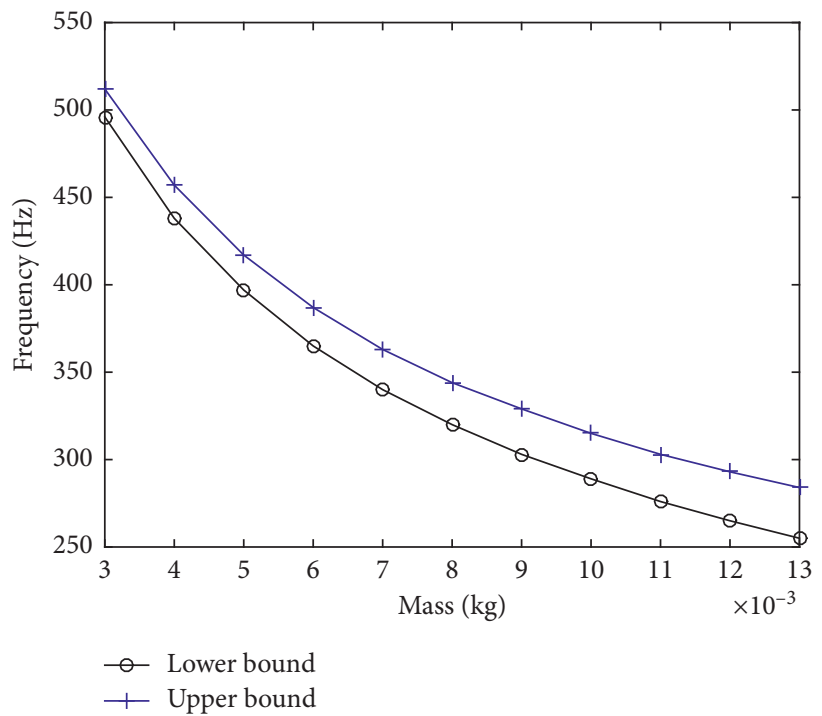

Figure 15: Lower and upper bounds vs. square mass.

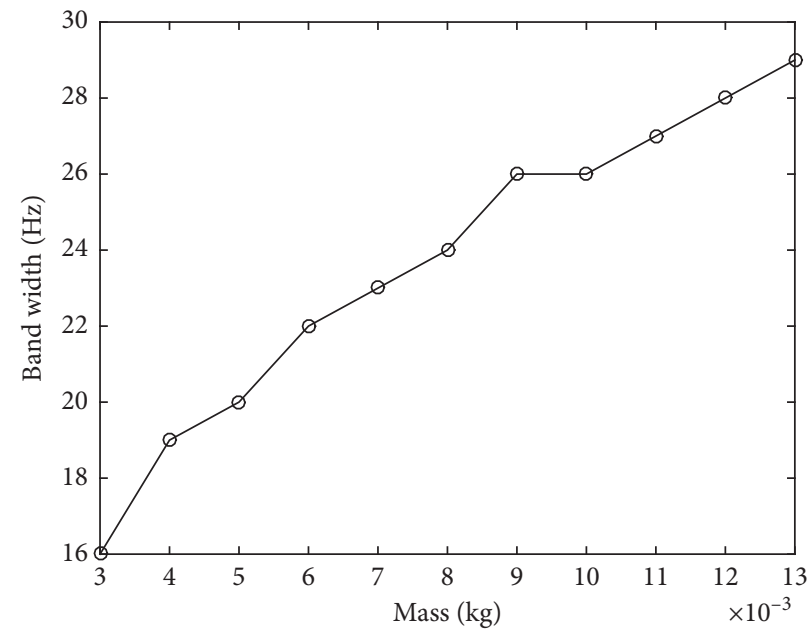

Figure 16: Bandwidth vs. square mass. 


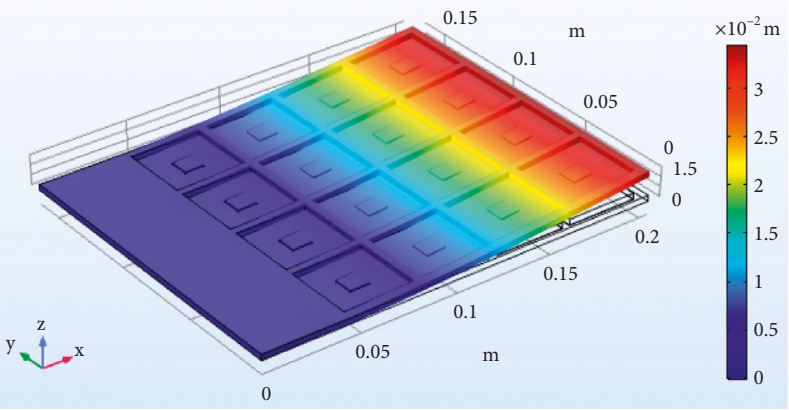

(a)

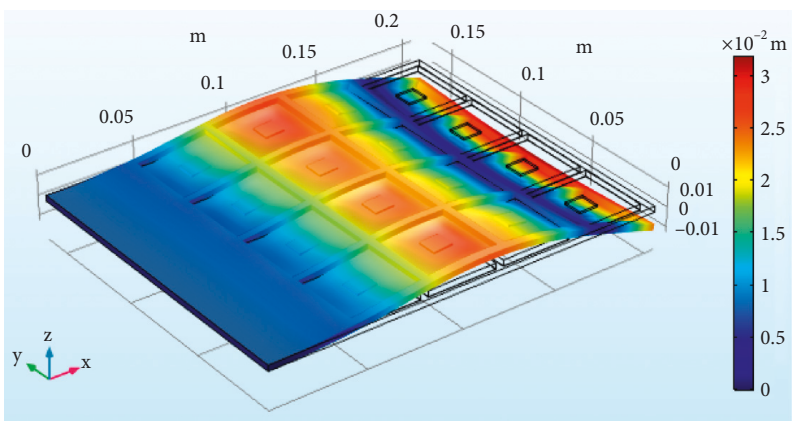

(c)

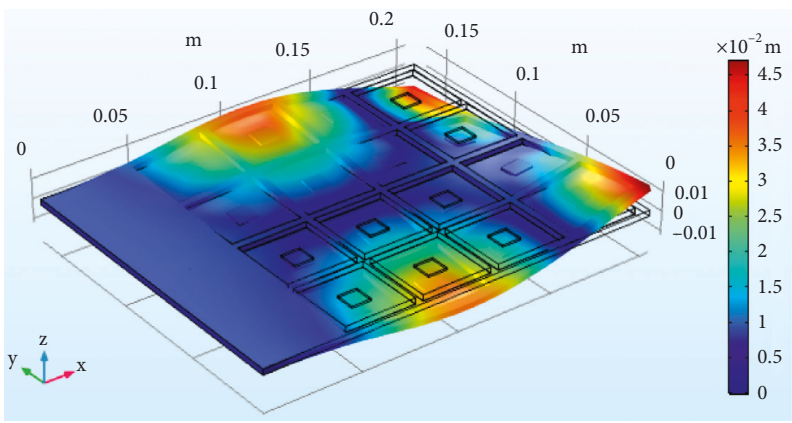

(e)

FIgURE 17: First six vibration mode shapes. (a) First. (b) Second. (c) Third. (d) Fourth. (e) Fifth. (f) Sixth.

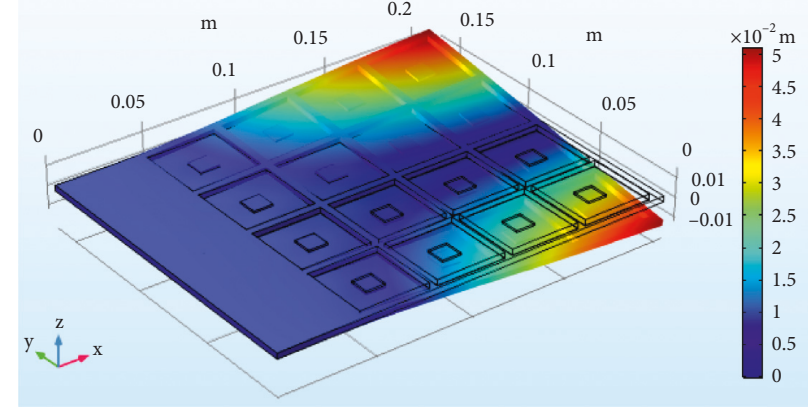

(b)

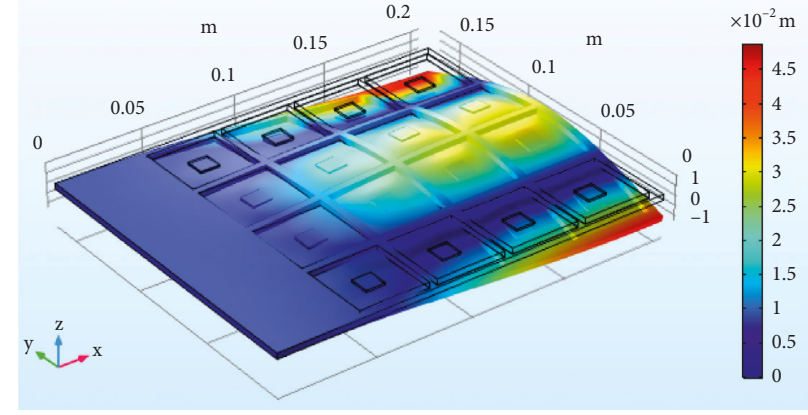

(d)

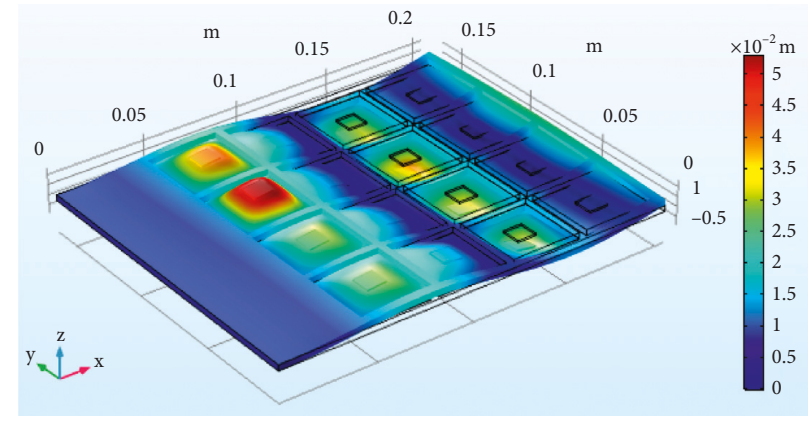

(f) end, vibration propagation functions at three response locations are calculated and plotted in Figures 20 22. It can be seen from the three figures that vibrations among [250, 370] $\mathrm{Hz}$ (shaded area) are strongly attenuated. That is to say, these vibrations cannot almost propagate along the PMP-LR within this frequency band and the bandwidth is equal to $120 \mathrm{~Hz}$. Thus, we can conclude that the measured bandgap approximates to $[250,370] \mathrm{Hz}$, and its bandwidth is about $120 \mathrm{~Hz}$. Also, we can see that the bandgaps measured from the three responses are almost the same because the bandgap is just an intrinsic property of the PMP-LR and independent of the source location. Based on the simulated results in Table 3, the bandgap is $[223,328] \mathrm{Hz}$ and its width is $106 \mathrm{~Hz}$. Therefore, we can conclude that experimental results are almost consistent with simulated ones. Bias may be generated by the following factors. Firstly, experimental conditions are not ideal. Secondly, the experimental PMP-LR is just a finitely periodic structure, while bandgaps are estimated based on an infinitely periodic structure.

\section{Conclusions}

This paper studied one kind of two-dimensional (2D) PMPLR for structural vibration energy harvesting, and vibrations are localized in some unit cells. In this case, energy loss can be reduced greatly. According to the energy balance theorem, the proposed structure will be better than existing systems. Firstly, an electromechanically coupled analytical model was built based on the Kirchhoff plate theory, and then modal analysis was done by using the Rayleigh-Ritz method. Next, effects of geometric and material parameters on vibration bandgaps were analyzed by finite elementbased simulations. Finally, experiments were carried out to validate the simulated results. Main conclusions of this paper include the following. (i) Energy localizations appear in vibration shapes of PMP-LR, which means that vibration energy can concentrate on some unit cells due to local resonators. (ii) Geometric configuration of the local resonator has direct effects on the frequency location of bandgap, while little effect on its width. In particular, the frequency 


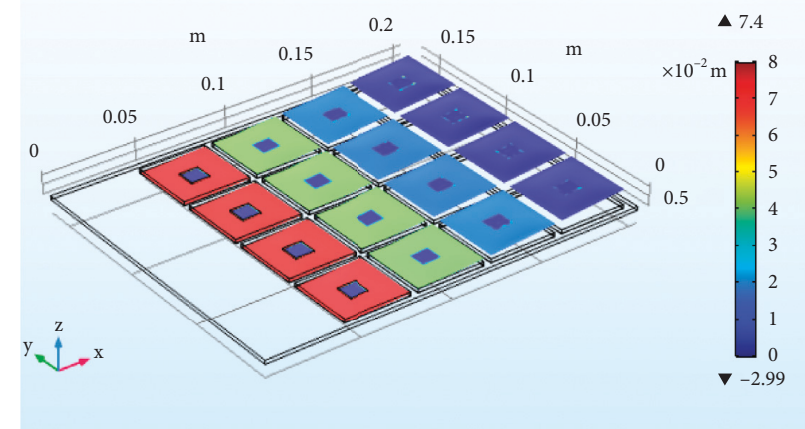

(a)

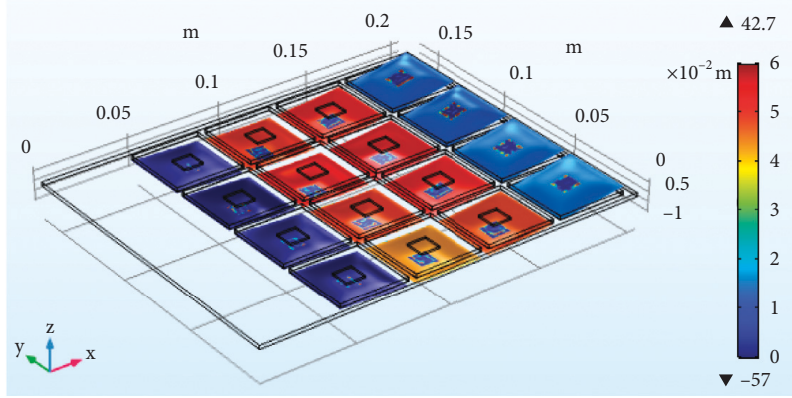

(c)

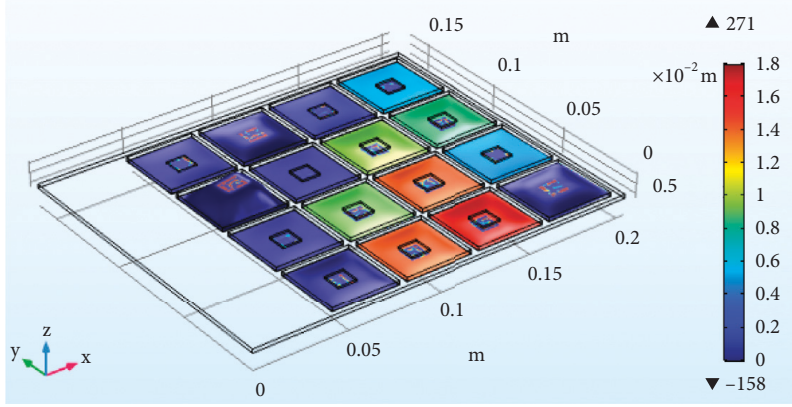

(e)

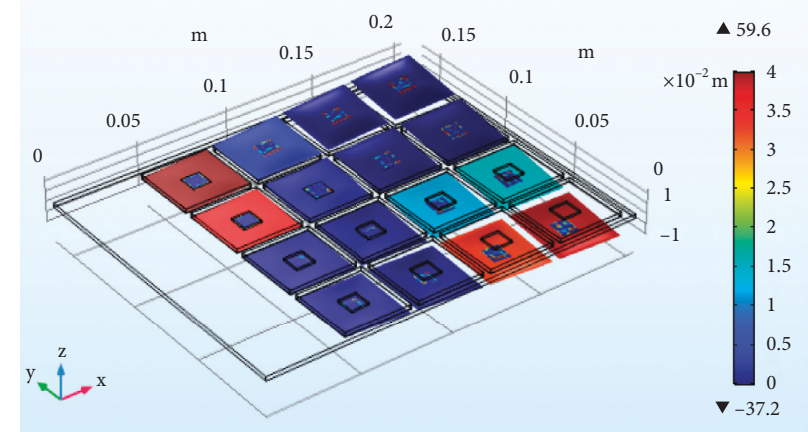

(b)

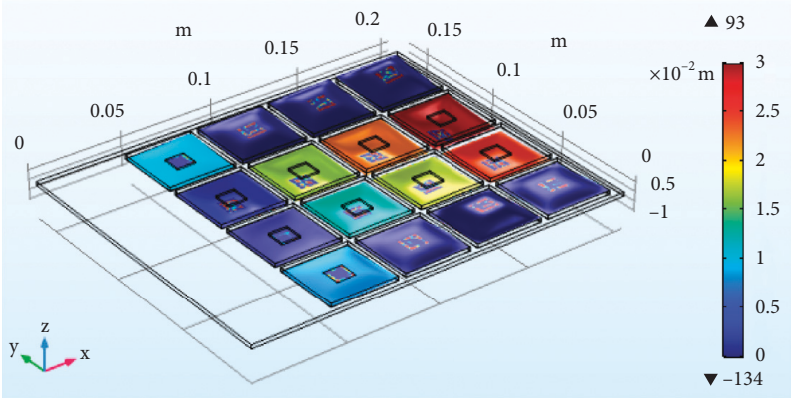

(d)

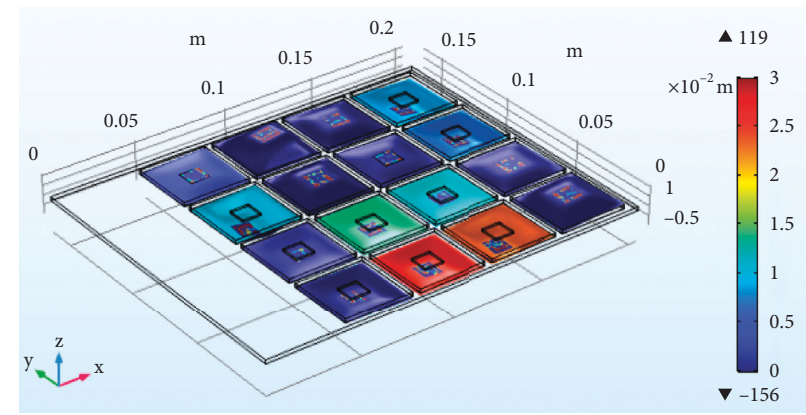

(f)

Figure 18: Piezoelectric voltages under the first six modes. (a) First. (b) Second. (c) Third. (d) Fourth. (e) Fifth. (f) Sixth.

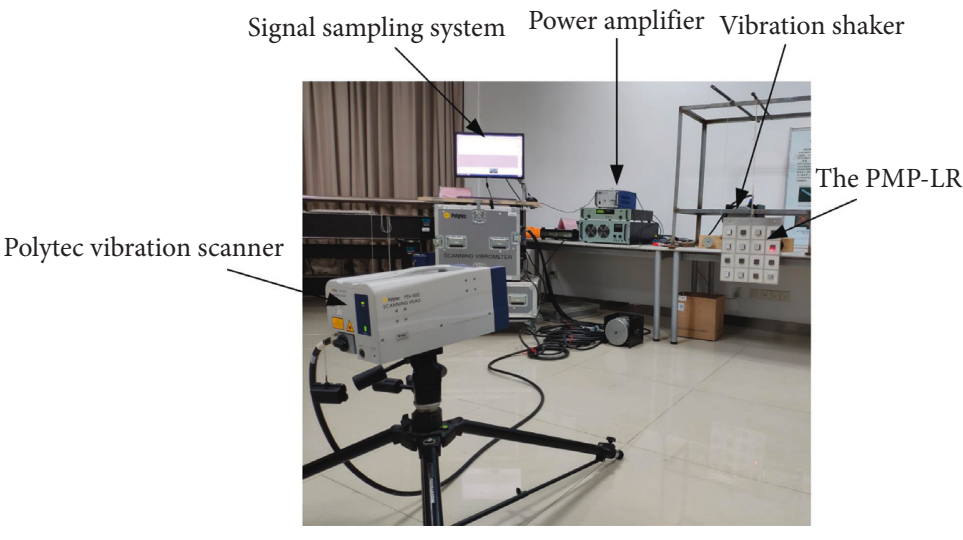

(a)

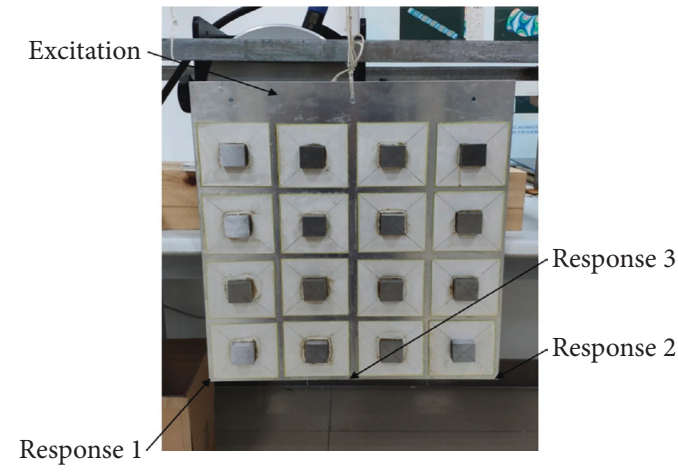

(b)

Figure 19: Experimental setup. (a) Whole testing system. (b) PMP-LR. 


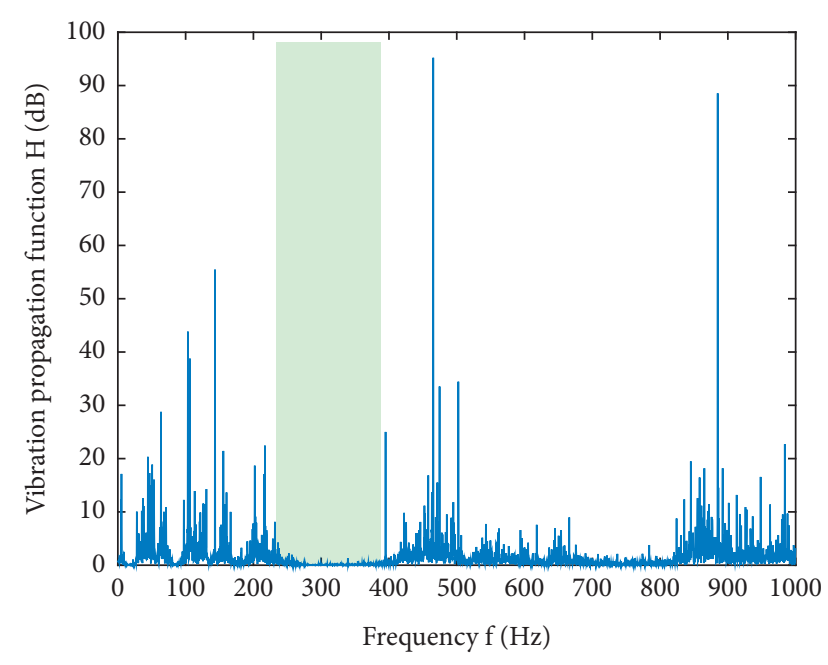

FIGURE 20: Vibration propagation function of response 1.

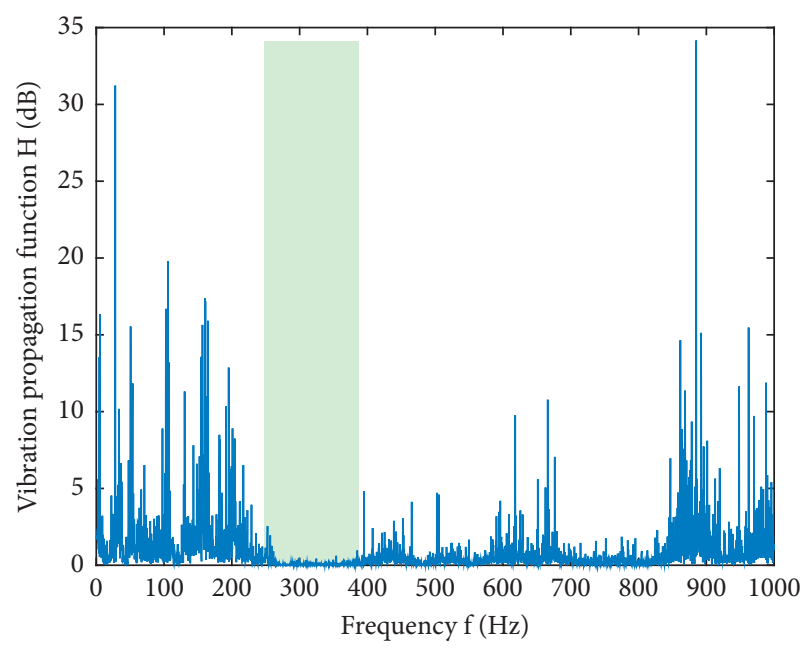

Figure 21: Vibration propagation function of response 2.

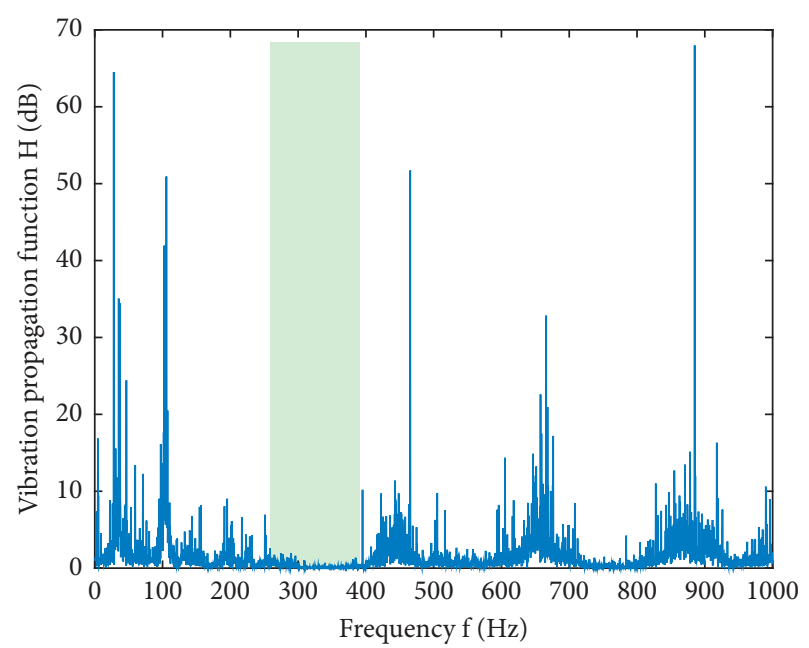

FIGURE 22: Vibration propagation function of response 3. location of bandgap is the lowest, and its width is the largest under mode 3. (iii) Both increasing the length of PZT-5H and decreasing the thickness of PZT-5H can make the vibration bandgap shift to low-frequency regions under a given lattice constant. At the same time, there exist optimal length and thickness of PZT-5H to obtain the maximum bandwidth. (iv) Increasing the mass can make the vibration bandgap shift to low-frequency regions and increase the band width. In future, more experiments need to be done to prove the theoretic results. Also, for structural vibration energy harvesting in practice, interface circuits should also be considered. Thus, an electromechanical model will be built and analyzed by integrating interface circuits [19], such as synchronized switching harvesting on an inductor interface circuits [20]. In this case, the design of the unit cell can be further optimized by considering all the aspects towards energy harvesting purpose, and optimal coupling coefficient can be obtained to increase the conversion efficiency. Moreover, topology optimization will be a promising method to optimize the layout of the unit cell for the most efficient energy harvesting [21], including the type of piezoelectric material (soft, hard, or else). Meanwhile, we will make a comparison with existing systems based on experiments.

\section{Data Availability}

The data used to support the findings of this study are available from the corresponding authors upon request.

\section{Conflicts of Interest}

The authors declare that there are no conflicts of interest regarding the publication of this paper.

\section{Acknowledgments}

This work was supported by the National Natural Science Foundations of China (grant nos. 51577189, 11772123, and 11702306) and the Royal Society International Exchange Scheme, China (grant no. IEC\NSFC\181462).

\section{References}

[1] C. Y. Liu, J. Teng, and N. Wu, "A wireless strain sensor network for structural health monitoring," Shock and Vibration, vol. 2015, Article ID 740471, 13 pages, 2015.

[2] K. S. Faisal and Z. S. Zeadally, "Energy harvesting in wireless sensor networks: a comprehensive review," Renewable and Sustainable Energy Reviews, vol. 55, no. 3, pp. 1041-1054, 2016.

[3] J. A. Paradiso and T. Starner, "Energy scavenging for mobile and wireless electronics," IEEE Pervasive Computing, vol. 4, no. 1, pp. 18-27, 2005.

[4] S. Zhou and L. Zuo, "Nonlinear dynamic analysis of asymmetric tristable energy harvesters for enhanced energy harvesting," Communications in Nonlinear Science and Numerical Simulation, vol. 61, no. 8, pp. 271-284, 2018.

[5] H. A. Sodano, D. J. Inman, and G. Park, "A review of power harvesting from vibration using piezoelectric materials," The Shock and Vibration Digest, vol. 36, no. 3, pp. 197-205, 2004. 
[6] S. R. Anton and H. A. Sodano, "A review of power harvesting using piezoelectric materials (2003-2006)," Smart Materials and Structures, vol. 16, no. 3, pp. R1-R21, 2007.

[7] S. Lee and B. D. Youn, "A design and experimental verification methodology for an energy harvester skin structure," Smart Materials and Structures, vol. 20, no. 5, article 057001, 2011.

[8] H. Yoon, B. D. Youn, and H. S. Kim, "Kirchhoff plate theorybased electromechanically-coupled analytical model considering inertia and stiffness effects of a surface-bonded piezoelectric patch," Smart Materials and Structures, vol. 25, no. 2, article 025017, 2016.

[9] A. Erturk, "Piezoelectric energy harvesting for civil infrastructure system applications: moving loads and surface strain fluctuations," Journal of Intelligent Material Systems and Structures, vol. 22, no. 17, pp. 1959-1973, 2011.

[10] U. Aridogan, I. Basdogan, and A. Erturk, "Analytical modeling and experimental validation of a structurally integrated piezoelectric energy harvester on a thin plate," Smart Materials and Structures, vol. 23, no. 4, article 045039, 2014.

[11] S. Gonella, A. C. To, and W. K. Liu, "Interplay between phononic bandgaps and piezoelectric microstructures for energy harvesting," Journal of the Mechanics and Physics of Solids, vol. 57, no. 3, pp. 621-633, 2009.

[12] Z. Chen, Y. Yang, Z. Lu, and Y. Luo, "Broadband characteristics of vibration energy harvesting using one-dimensional phononic piezoelectric cantilever beams," Physica B: Condensed Matter, vol. 410, pp. 5-12, 2013.

[13] L. Y. Wu, L. W. Chen, and C. M. Liu, "Acoustic energy harvesting using resonant cavity of a sonic crystal," Applied Physics Letter, vol. 95, no. 1, article 013506, 2009.

[14] Z. Liu, X. X. Zhang, Y. W. Mao et al., "Locally resonant sonic materials," Science, vol. 289, no. 5485, pp. 1734-1736, 2000.

[15] C. Sugino, S. Leadenham, M. Ruzzene, and A. Erturk, "An investigation of electroelastic bandgap formation in locally resonant piezoelectric metastructures," Smart Materials and Structures, vol. 26, no. 5, article 055029, 2017.

[16] N. Kherraz, L. Haumesser, F. Levassort, P. Benard, and B. Morvan, "Hybridization bandgap induced by an electrical resonance in piezoelectric metamaterial plates," Journal of Applied Physics, vol. 123, no. 9, article 094901, 2018.

[17] L. Raghavan and A. S. Phani, "Local resonance bandgaps in periodic media: theory and experiment," Journal of the Acoustical Society of America, vol. 134, no. 3, pp. 1950-1959, 2013.

[18] A. Bergamini, T. Delpero, L. D. Simoni, L. D. Lillo, M. Ruzzene, and P. Ermanni, "Phononic crystal with adaptive connectivity," Advanced Materials, vol. 26, no. 9, pp. 13431347, 2014.

[19] G. Hu, B. Zhao, L. Tang, J. Liang, and R. Das, "Modeling of partially covered piezoelectric energy harvester connected to AC-DC interface circuit," in Proceedings of the International Conference on Noise and Vibration Engineering, Leuven, Belgium, September 2018.

[20] Z. Chen, J. He, J. Liu, and Y. Xiong, "Switching delay in selfpowered nonlinear piezoelectric vibration energy harvesting circuit: mechanisms, effects, and solutions," IEEE Transactions on Power Electronics, vol. 34, no. 3, pp. 2427-2440, 2019.

[21] C. J. Rupp, A. Evgrafov, K. Maute, and M. L. Dunn, "Design of piezoelectric energy harvesting systems: a topology optimization approach based on multilayer plates and shells," Journal of Intelligent Material Systems and Structures, vol. 20, no. 16, pp. 1923-1939, 2009. 


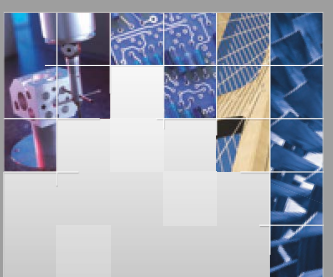

\section{Enfincering}
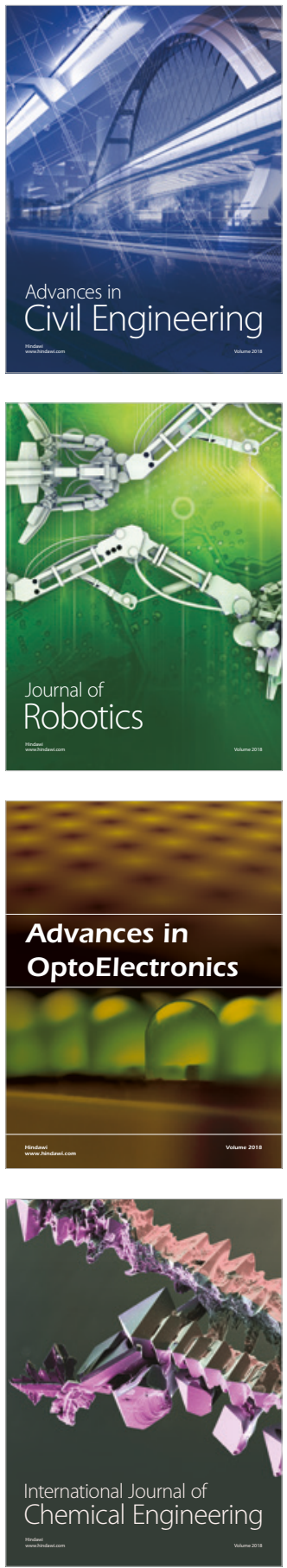

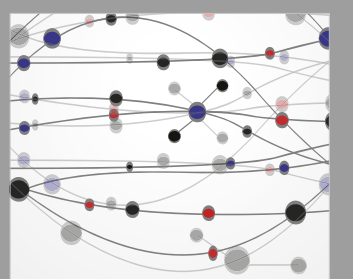

\section{Rotating \\ Machinery}

The Scientific World Journal

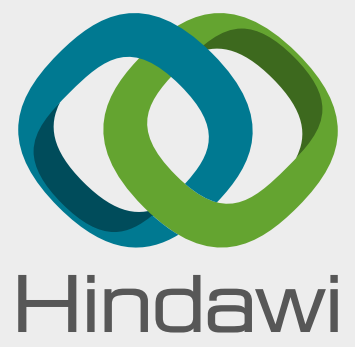

Submit your manuscripts at

www.hindawi.com
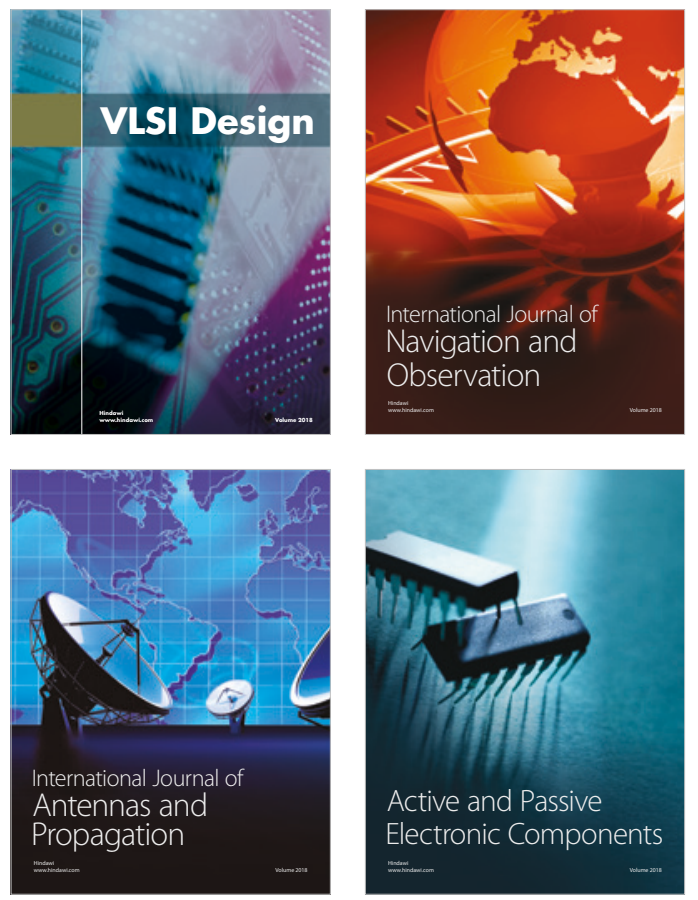
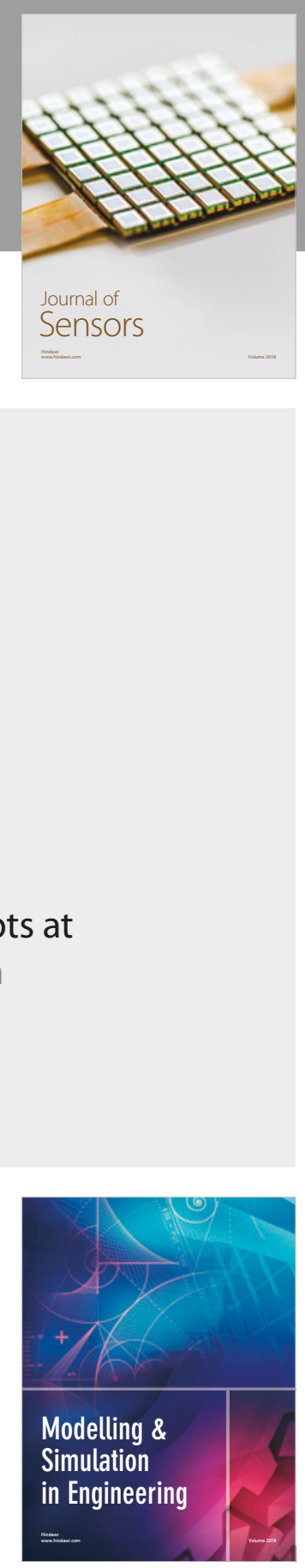

\section{Advances \\ Multimedia}
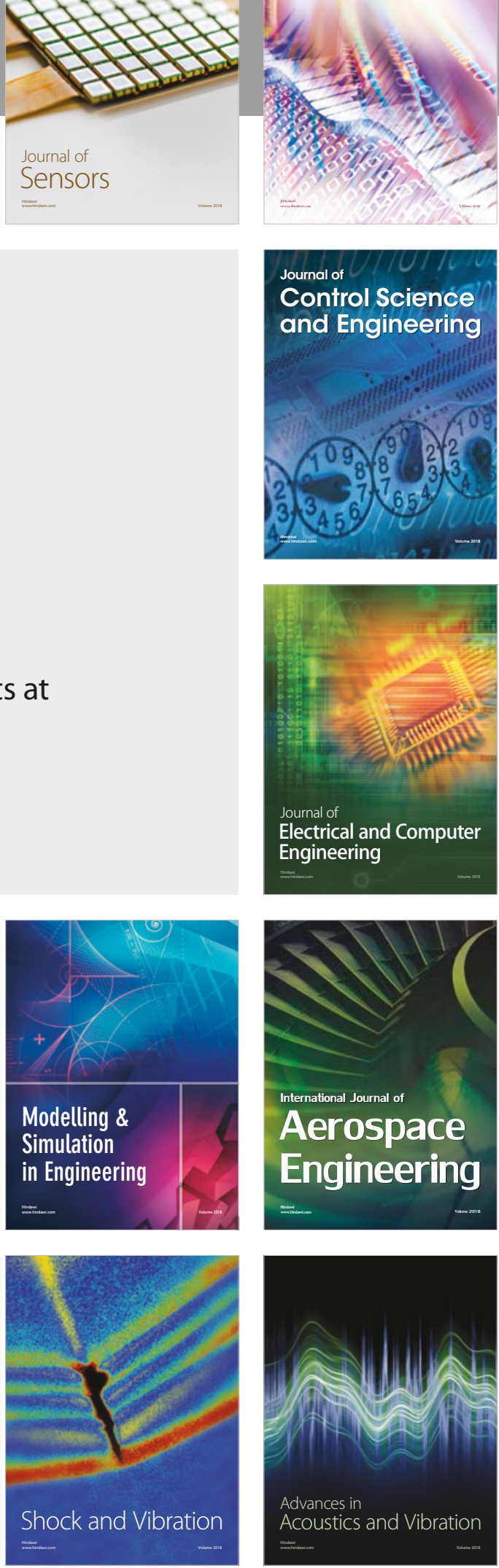